\title{
Bayesian inference for fault slip distributions based on ensemble modeling of the uncertainty of underground structure
}

\author{
Ryoichiro Agata $^{1}$, Amato Kasahara ${ }^{2}$, Yuji Yagi ${ }^{3}$ \\ ${ }^{1}$ Japan Agency for Marine-Earth Science and Technology, \\ 3173-25 Showa-machi, Kanazawa-ku, Yokohama 2360001, Japan. Email: agatar@jamstec.go.jp \\ ${ }^{2}$ Independent researcher \\ ${ }^{3}$ Faculty of Life and Environmental Sciences, University ofTsukuba, Tsukuba, Ibaraki 305-8572, Japan.
}

Received XXX; in original form XXX

\section{SUMMARY}

The prediction errors that originate from the uncertainty of underground structure is often a major contributor of the errors between the data and the model predictions in fault slip estimation using geodetic or seismic waveform data. However, most studies on slip inversions either neglect the model prediction errors or do not distinguish them from observation errors. Several methods that explicitly incorporated the model prediction errors in slip estimation, which has been proposed in the past decade, commonly assumed a Gaussian distribution for the stochastic property of the prediction errors to simplify the formulation. Moreover, the information on slip distribution and the underground structure is expected to be successfully extracted from the data by accurately incorporating the stochastic property of the prediction errors. In this study, we develop a novel flexible Bayesian inference method for estimating fault slips that can accurately incorporate non-Gaussian prediction errors. This method considers the uncertainty of the underground structure, including fault geometry based on the ensemble modeling of the uncertainty of Green's function. Furthermore, the framework allows the estimation of the posterior probability density function (PDF) of the parameters of the underground struc- 
ture, by calculating the likelihood of each sample in the ensemble. To validate the advantage of the proposed method, we performed simple numerical experiments for estimating the slip deficit rate (SDR) distribution on a 2D thrust fault using synthetic data of surface displacement rates. In the experiments, the dip angle of the fault plane was the parameter used to characterize the underground structure. The proposed method succeeded in estimating a posterior PDF of SDR that is consistent with the true one, despite the uncertain and inaccurate information of the dip angle. In addition, the method could estimate a posterior PDF of the dip angle that has a strong peak near the true angle. In contrast, the estimation results obtained using a conventional approach, which introduces regularization based on smoothing constraints and does not explicitly distinguish the prediction and observation errors, included a significant amount of bias, which was not noticed in the results obtained using the proposed method. The experiments with different settings of the parameters suggested that inaccurate prior information of the underground structure with a small variance possibly results in significant bias in the estimation results, particularly the posterior PDFs for SDR, those for the underground structure, and the posterior predicted PDF of the displacement rates. The distribution shapes of the prediction errors for the representative model parameters in certain observation points are significantly asymmetric with large absolute values of the sample skewness, for which Gaussian approximation is not usually applied.

Key words: Inverse theory, Probability distributions, Earthquake source observations

\section{INTRODUCTION}

Estimation of fault slip distributions, including co-seismic slips, aseismic slips, and interplate couplings, is an essential step to better understand the earthquake generation processes and assess seismic hazards. For estimating fault slip distributions using geodetic or waveform data, the underground fault slip parameters typically are linearly related to the surface geodetic or seismic response in each observation point using a coefficient matrix calculated based on Green's function, assuming linear elastic media. Green's function is characterized by the setting of the numerical model for the underground structure (i.e., seismic velocity structure and fault geometry). The unknown fault slip parameters and the parameters for the underground structure can be considered 
as "model parameters" and "latent parameters", respectively, in terms of general discussion on inverse problems. The latent parameters are "latent" because they are neither model parameters nor the observables, but play a role in the stochastic model (Bishop 2006).

In real-world problems, the value of Green's function is uncertain because the corresponding underground structure is always uncertain. Because of this uncertainty, the surface responses that are predicted by combining the fault slip parameters and Green's function are always associated with prediction errors relative to the true response. Although ignoring the prediction errors in the inverse problem formulations due to the uncertainty in Green's function possibly introduces bias into the estimation results, majority of the studies still neglect it or do not distinguish it from observation errors in the inverse problem formulations. However, the past decade has seen remarkable progress in the mathematical formulation of the prediction errors due to the uncertainty in Green's function in earthquake source inversions. The pioneering work of Yagi \& Fukahata (2011) proposed an inversion scheme that introduces the error of Green's function that follows a Gaussian distribution and iteratively estimated the model parameters and the covariance matrix for the prediction errors simultaneously. Minson et al. (2013) introduced a Gaussian model for describing the prediction errors, using a diagonal covariance matrix with variances that are positively correlated with the observed amplitudes. Duputel et al. (2014) proposed a comprehensive framework to compute the covariance matrix for the prediction errors based on uncertain and presumably inaccurate prior knowledge of the underground structure. Both Minson et al. (2013) and Duputel et al. (2014) claimed that available fault slip models for the same earthquake are often quite contrasting, because of the combination of an inaccurate forward model and subjective regularization of the inverse problem. They tried to resolve this problem based on fully Bayesian inference by developing an accurate stochastic model that better describes the modeling uncertainty in predicting the response at the observation points for geodetic and seismic waveform data. These studies were additionally followed by certain recent efforts to include the prediction errors explicitly in the inversion schemes (Hallo \& Gallovič 2016; Ragon et al. 2018; Agata 2020).

The common feature of the methods that were proposed in the referred studies herein is that the prediction errors are assumed to follow the Gaussian distribution. The use of Gaussian dis- 
tribution makes the analysis of equations simple that is necessary for considering the combined effect of the prediction errors and observation errors. The assumption of Gaussianity may be valid when the assumed deviation of the latent parameters is so small that the relationship between the predicted response and the parameters are well approximated by linearity (Duputel et al. 2014). However, it is difficult to make sure that this approximation has acceptable accuracy for all target problems. Moreover, these studies do not extract the underground structure information from the observation data. Accurately incorporating the stochastic property of the prediction errors in fault slip estimations may help in obtaining the underground structure information in the data contains as well.

In the studies of data assimilation, the introduction of ensemble modeling of probability density functions (PDFs) increased the generality of sequential filtering, which can be interpreted as a type of an on-line inversion method. For example, in the ensemble Kalman filter (EnKF) (Evensen 1994), stochastic variables that are necessary to be evaluated are approximately calculated based on an ensemble of candidate models, which enable the idea of Kalman filtering to apply to nonlinear and larger-scale problems. Particle filter (Gordon et al. 1993; Kitagawa 1993, 1996) generates many candidate models (particles) that are sampled from the model space. The method allows for further flexible modeling by choosing the candidates that are closer to the true model based on the likelihood of each candidate. These methods, based on ensemble modeling of PDFs, are widely used in the last few decades with the help of an increasing amount of computation resources. The fault slip estimation methods in the next generation can be designed by considering the techniques of approximating the target PDF using an ensemble of sample models, narrowing down the model space by choosing candidate models from the ensemble, and incorporating realistic understructure models when generating the ensemble. Yamaguchi et al. (2017) is an example of introducing stochastic modeling based on an ensemble of candidate underground structure models for fault slip estimation. This study calculated 1,000 coefficient matrices based on 1,000 different seismic velocity models and performed inversion analyses 1,000 times using each of the coefficient matrices. The stochastic properties of the 1,000 slip estimation results were then analyzed. The result of 
this study indicates that the use of the ensemble approach possibly applies to not only the simple problems but also those based on recent, more sophisticated forward modeling methods.

This study considers the uncertainty of the underground structure based on ensemble modeling of the Green's function uncertainty in estimating fault slip distributions. Such an approach allows for the (1) consideration of the prediction error more accurately without assuming Gaussianity and (2) extraction of information on not only fault slips but also the underground structure from the data. We set a Bayesian model and draw samples from the posterior PDF of the fault slip using a type of the Markov chain Monte Carlo method, in which we calculate an integration term that combines the terms of the observation and the prediction errors using the Monte Carlo approximation. This approach enables the estimation of the posterior PDF of the slip distribution, accurately incorporating the stochastic property of the prediction errors without assuming Gaussianity. Moreover, because the likelihood for each coefficient matrix is also calculated in the process of performing the Monte Carlo integration, we can simultaneously find underground structure models that are consistent with the observation data. Furthermore, accurate incorporation of the stochastic property of the prediction errors in a fully Bayesian framework encourages slip estimations without the regularization of smoothing constraints as a prior PDF, as pointed out by Minson et al. (2013) and Duputel et al. (2014). The framework proposed in this study is generally applicable to inverse problems that include latent parameters for which only uncertain information is available, similar to the majority of the geophysical inverse problems.

This paper is organized as follows: Section 2 describes the formulation of Bayesian inference fault slips based on ensemble modeling of underground structure uncertainty. Section 3 presents a method for estimating the parameters of the underground structure. In Section 4, the proposed method is applied to a simple two-dimensional (2D) synthetic test of a geodetic inverse problem. Finally, Section 6 provides the concluding remarks. 


\section{FULLY BAYESIAN FORMULATION OF FAULT SLIP ESTIMATIONS BASED ON ENSEMBLE MODELING OF THE UNDERGROUND STRUCTURE UNCERTAINTY}

\subsection{Observation and prediction errors in Bayesian formulation of fault slip estimations}

Let us consider an estimation problem of $\mathbf{m}$, a vector for the parameters of slip distribution, or model parameters in a more general viewpoint, using $\mathbf{d}$, a vector for the observation data. Hereafter, we interchangeably use "fault slip parameters" and "model parameters." This estimation problem can be formulated as a Bayesian source inversion, in which we estimate the posterior PDF of $\mathbf{m}$, as

$P(\mathbf{m} \mid \mathbf{d})=\kappa P(\mathbf{d} \mid \mathbf{m}) P(\mathbf{m})$

where $P(\mathbf{m})$ is a prior PDF of the model parameters, and the conditional PDF $P(\mathbf{d} \mid \mathbf{m})$ and $P(\mathbf{m} \mid \mathbf{d})$ are a likelihood function and a posterior PDF of the model parameters, respectively. $\kappa=1 / P(\mathbf{d})$ is a normalization factor, which takes a constant value because the observation data take fixed values. The prior PDF is set based on our prior knowledge of the slip distribution. In addition, a properly defined likelihood function is required for evaluating the posterior PDF. In defining the likelihood function, we relate the data and the model parameters in two steps. First, the surface displacement is predicted by a function $\boldsymbol{g}(\mathbf{m})$. Herein, we consider a linear system, where the prediction is simply made by matrix-vector multiplication, i.e., $\boldsymbol{g}(\mathbf{m})=\mathbf{G m}$, where $\mathrm{G}$ is a coefficient matrix calculated based on Green's function. We denote the predicted response in the observation point by $\mathbf{d}_{\text {pred }}$. The stochastic property of the model prediction for a given $\mathbf{m}$, which we call the prediction errors, is described by a conditional PDF $P\left(\mathbf{d}_{\text {pred }} \mid \mathbf{m}\right)$. The stochastic property of the measurement of the response, which is characterized by the knowledge of the error property of the observation instrument, is denoted by a conditional PDF $P\left(\mathbf{d} \mid \mathbf{d}_{\text {pred }}\right)$. The likelihood function can then be obtained by marginalizing the product of these two PDFs with $\mathbf{d}_{\text {pred }}$ (Duputel et al. 2014), as

$P(\mathbf{d} \mid \mathbf{m})=\int P\left(\mathbf{d} \mid \mathbf{d}_{\text {pred }}\right) P\left(\mathbf{d}_{\text {pred }} \mid \mathbf{m}\right) d \mathbf{d}_{\text {pred }}$ 
If we assume that both PDFs follow the Gaussian distribution, the integration of Equation 2 can be calculated analytically. The previous studies (e.g., Duputel et al. (2014); Ragon et al. (2018)) made use of this fact and assumed that the prediction errors follow the Gaussian distribution to make the formulation and calculation simple and efficient. In contrast, the proposed approach calculates this integration based on the Monte Carlo integration using random samples of $\mathbf{d}_{\text {pred }}$, without assuming Gaussianity in the prediction errors.

\subsection{Calculation of likelihood function based on ensemble modeling of Green's function uncertainty}

We assumed that the main source of the prediction errors is the uncertainty of the underground structure, characterized by the latent parameters that characterize the fault geometry and elastic properties, which are denoted by $\varphi$. Hereafter, we interchangeably use "parameters for the underground structure" and "latent parameters." We suppose that the $P(\varphi)$, the stochastic property of the latent parameters, which is also interpreted as the prior PDF for the latent parameters, is already provided based on the existing underground structure models. We draw a significant number of random samples $\varphi^{(n)}$ from $P(\varphi)$. Because the coefficient matrix is determined when $\varphi$ is fixed, we can produce an ensemble of the matrices consisting of $\mathbf{G}\left(\varphi^{(n)}\right)$, where $n=1, \ldots, N$ and $N$ is the ensemble size for the random samples. Herein, $\mathbf{G}\left(\boldsymbol{\varphi}^{(n)}\right)$ means that the coefficient matrix is a (nonlinear) function of the latent parameters. We can then straightforwardly calculate the ensemble of $\mathbf{d}_{\text {pred }}$, as

$\left\{\mathbf{d}_{\text {pred }}^{(1)}(\mathbf{m}), \ldots, \mathbf{d}_{\text {pred }}^{(N)}(\mathbf{m})\right\}$, where $\mathbf{d}_{\text {pred }}^{(n)}(\mathbf{m})=\mathbf{G}\left(\boldsymbol{\varphi}^{(n)}\right) \mathbf{m}(n=1, \ldots, N)$.

$\mathbf{d}_{\text {pred }}^{(n)}(\mathbf{m})$ s can be considered as random samples from $P\left(\mathbf{d}_{\text {pred }} \mid \mathbf{m}\right)$. Subsequently, Monte Carlo integration can be used to approximate the integration in Equation 2 based on the ensemble of a sufficiently large size $N$, as

$P(\mathbf{d} \mid \mathbf{m}) \simeq \frac{1}{N} \sum_{n=1}^{N} P\left(\mathbf{d} \mid \mathbf{d}_{\text {pred }}^{(n)}(\mathbf{m})\right)$.

For $P\left(\mathbf{d} \mid \mathbf{d}_{\text {pred }}\right)$, it is natural to assume a Gaussian distribution in most of the cases, as

$P\left(\mathbf{d} \mid \mathbf{d}_{\text {pred }}\right)=(2 \pi)^{-N / 2}\|\mathbf{E}\|^{-1 / 2} \exp \left[-\frac{1}{2}\left(\mathbf{d}-\mathbf{d}_{\text {pred }}\right)^{\mathrm{T}} \mathbf{E}^{-1}\left(\mathbf{d}-\mathbf{d}_{\text {pred }}\right)\right]$, 
where $\mathbf{E}$ is the covariance matrix that is determined based on the error characteristics of the observation instruments and the data processing. When we assume such an analytical form of $P\left(\mathbf{d} \mid \mathbf{d}_{\text {pred }}\right)$, we can calculate Equation 4 . Subsequently, we can approximately evaluate the posterior $\mathrm{PDF}$ as

$$
\begin{aligned}
P(\mathbf{m} \mid \mathbf{d}) & =\kappa P(\mathbf{d} \mid \mathbf{m}) P(\mathbf{m}) \\
& \simeq \kappa \frac{1}{N} \sum_{n=1}^{N} P\left(\mathbf{d} \mid \mathbf{d}_{\text {pred }}^{(n)}(\mathbf{m})\right) P(\mathbf{m}) .
\end{aligned}
$$

To accurately perform the Monte Carlo integration, we need to take a sufficiently large ensemble size $N$, which may increase the associated calculation cost. However, the proposed method can fully account for the association in prediction errors by calculating many matrix-vector products. Consequently, when the number of observation data is increased $k$ times, the proposed method requires only $k$ times larger calculation cost compared to the original problem. This efficiency in time complexity is contrary to that for the previous methods based on the assumption of Gaussianity, in which the covariance matrix for prediction errors is a function of the model parameter. The calculation of the determinant of a different covariance matrix is required in each step of a sampling algorithm of the posterior PDF or an iterative optimization algorithm, which generally increases the computation cost $k^{3}$ times for a $k$ times larger number of observation data. As Kubo et al. (2016) mentioned, such a time complexity associated with the use of previously proposed approaches for incorporating the prediction errors may lead to enormous calculation costs when the parameter search is performed based on Bayesian sampling. Therefore, whether the proposed method is computationally more expensive than the methods based on Gaussian approximation of the prediction errors may depend on the nature of the problem.

\subsection{Sampling algorithm for the posterior probability distribution of fault slips}

We use the replica-exchange Monte Carlo method (REMC) (Swendsen \& Wang 1986; Geyer 1991), also known as parallel tempering, a type of the Markov chain Monte Carlo (MCMC) method (Metropolis et al. 1953), to perform the Bayesian sampling from the target posterior PDF. REMC is capable of drawing samples from the target PDF much more efficiently than simpler 
MCMC algorithms such as the Metropolis method (Metropolis et al. 1953), by performing several samplings with different PDFs characterized by varying "temperatures" in parallel, between which the samples are randomly exchanged every several time steps. To perform REMC, we use an algorithm that is similar to that used in Kano et al. (2017), a recent application example of the method to seismology. Algorithm 1 shows details of the algorithm. If $L$, the number of chains, is taken to be 1 , and the exchange of the samples is not carried out, this algorithm becomes identical to that of the Metropolis method. In our applications, the temperature is taken to be $T_{l}=T_{2}^{l-1}(l=1, \ldots, L)$, where $T_{1}=1$, which means $P_{1}(\mathbf{m})=P(\mathbf{m} \mid \mathbf{d}) . T_{2}$ and other constants used in Algorithm 1 are adjusted through trial and error so that the target PDF is sampled properly.

\section{ESTIMATION OF THE POSTERIOR PDF OF THE LATENT PARAMETERS FOR THE UNDERGROUND STRUCTURE}

In the previous Section, only the posterior PDF of the model parameters is estimated by using the observation data. However, the data may also contain the information of the latent parameter. Herein, we discuss the estimation of the posterior PDF of the latent parameters regarding an underground structure in the post-process of Bayesian sampling of the model parameters. Strictly speaking, the phrasing "estimation of the latent parameter" contradicts its definition; however, we use the "latent parameter" for denoting the parameters of the underground structure to distinguish them from the model parameters of the slip distribution.

\subsection{Interpretation of the proposed algorithm in the view of the estimation of joint probability for the model and latent parameters}

Before discussing the posterior PDF for the latent parameters, we consider the joint posterior PDF of the model or slip parameters and the latent parameters of the underground structure after obtaining the data, which can be formulated based on Bayes' theorem as

$P(\mathbf{m}, \boldsymbol{\varphi} \mid \mathbf{d})=\kappa P(\mathbf{d} \mid \mathbf{m}, \boldsymbol{\varphi}) P(\mathbf{m}, \boldsymbol{\varphi})$ 
For simplicity, we hereafter consider a case where the prior information of $\mathbf{m}$ and $\varphi$ are independent, i.e., $P(\mathbf{m}, \boldsymbol{\varphi})=P(\mathbf{m}) P(\boldsymbol{\varphi})$, although the applicability of the method is not limited to such a case. The marginal posterior PDF of $\mathbf{m}$ can be obtained by marginalizing Equation 8 with $\varphi$ as

$P(\mathbf{m} \mid \mathbf{d})=\kappa \int P(\mathbf{d} \mid \mathbf{m}, \boldsymbol{\varphi}) P(\mathbf{m}) P(\boldsymbol{\varphi}) d \boldsymbol{\varphi}$

If we draw random samples $\varphi^{(n)}$ from $P(\boldsymbol{\varphi})$, Equation 9 can be approximately evaluated based on Monte Carlo integration as

$$
\begin{aligned}
P(\mathbf{m} \mid \mathbf{d}) & =\kappa \int P(\mathbf{d} \mid \mathbf{m}, \boldsymbol{\varphi}) P(\mathbf{m}) P(\boldsymbol{\varphi}) d \boldsymbol{\varphi} \\
& \simeq \kappa \frac{1}{N} \sum_{n=1}^{N} P\left(\mathbf{d} \mid \mathbf{m}, \boldsymbol{\varphi}^{(n)}\right) P(\mathbf{m}) .
\end{aligned}
$$

By comparing this equation with Equation 7, we find that $P\left(\mathbf{d} \mid \mathbf{d}_{\text {perf }}^{(n)}(\mathbf{m})\right)=P\left(\mathbf{d} \mid \mathbf{m}, \varphi^{(n)}\right)$. In Section 2, we presented the proposed algorithm from the viewpoint of the mathematical formulation of the prediction errors. However, the same algorithm can also be interpreted as the marginalization of a posterior joint probability using the latent parameters. The latter interpretation is useful to derive the formula for estimating the posterior PDF of the latent parameters.

\subsection{Calculation of the posterior PDF of the latent parameters for an underground structure}

Further, we obtain the posterior PDF of $\varphi$ by marginalizing Equation 8 with $\mathbf{m}$ as

$P(\boldsymbol{\varphi} \mid \mathbf{d})=\kappa \int P(\mathbf{d} \mid \mathbf{m}, \boldsymbol{\varphi}) P(\mathbf{m}) P(\boldsymbol{\varphi}) d \mathbf{m}$

Suppose we have obtained $M\left(=M_{\text {iteration }}-M_{\text {burnin }}\right.$ using the constants defined in Algorithm 1) samples from $P(\mathbf{m} \mid \mathbf{d})$ using the sampling algorithm presented in Section 2.3. If we replace $P(\mathbf{m})$ in Equation 12 with a new $\operatorname{PDF} P_{\text {new }}(\mathbf{m})=P(\mathbf{m} \mid \mathbf{d})$ from which we obtained the samples $\left\{\mathbf{m}^{(1)}, \ldots, \mathbf{m}^{(M)}\right\}$, we can approximately calculate $P(\varphi \mid \mathbf{d})$ based on the Monte Carlo integration using the samples as

$$
\begin{aligned}
P(\boldsymbol{\varphi} \mid \mathbf{d}) & =\kappa \int P(\mathbf{d} \mid \mathbf{m}, \boldsymbol{\varphi}) P_{\mathrm{new}}(\mathbf{m}) P(\boldsymbol{\varphi}) d \mathbf{m} \\
& \simeq \kappa \frac{1}{M} \sum_{m=1}^{M} P\left(\mathbf{d} \mid \mathbf{m}^{(m)}, \boldsymbol{\varphi}\right) P(\boldsymbol{\varphi})
\end{aligned}
$$


$P(\boldsymbol{\varphi})$ can be approximated using the same $N$ samples of $\varphi$ as those used in Equation 3, 11 and others by, for example, particle approximation as

$\hat{P}(\boldsymbol{\varphi})=\frac{1}{N} \sum_{n=1}^{N} \delta\left(\boldsymbol{\varphi}-\boldsymbol{\varphi}^{(n)}\right)$.

By substituting this term into Equation 14, the marginal posterior PDF of $\varphi$ can be also written based on the particle approximation as

$\hat{P}(\boldsymbol{\varphi} \mid \mathbf{d})=\kappa \frac{1}{N} \sum_{n=1}^{N} w^{(n)} \delta\left(\varphi-\varphi^{(n)}\right)$

where

$w^{(n)}=\frac{1}{M} \sum_{m=1}^{M} P\left(\mathbf{d} \mid \mathbf{m}^{(m)}, \boldsymbol{\varphi}^{(n)}\right)$.

Equation 15 has the form of importance sampling, which is known to be more efficient in approximating a distribution than ordinary sampling methods. Because $P\left(\mathbf{d} \mid \mathbf{m}^{(m)}, \boldsymbol{\varphi}^{(n)}\right)$ is calculated when MCMC sampling for $P(\mathbf{m} \mid \mathbf{d})$ is performed, as evidently seen in Equation 11, we can readily calculate Equation 15. We herein present a particle approximation based on the delta function to approximate $P(\boldsymbol{\varphi} \mid \mathbf{d})$ for simplicity, which allows for the drawing of a histogram that represents $P(\varphi \mid \mathbf{d})$. Applying the kernel density estimation (Rosenblatt 1956) using a smoother kernel function such as the Gaussian function should produce an approximated PDF with a smoother distribution.

\subsection{Advantage over a naive estimation method of the joint probability of fault slip and underground structure}

Section 2 presented an algorithm for Bayesian sampling of the posterior PDF of the model parameters considering the prediction errors that originated from the uncertainty of the latent parameters for the underground structure. The discussion in this Section so far suggests that the proposed approach is mathematically equivalent to Bayesian sampling of the joint posterior probability of both the model and latent parameters using the prior PDF that describes the stochastic properties of the latent parameters and marginalizing the joint probability with the latent parameters afterward. The difference between the two approaches is whether to marginalize the target PDF before or after 
the sampling process. In the context of earthquake source inversions, such a close relationship between these two approaches suggests that simultaneous estimation of the parameters for fault slip and the underground structure (e.g., Fukuda \& Johnson (2010); Fukahata \& Wright (2008); Agata et al. (2018)) is closely related to fault slip inversion methods considering the prediction errors that originated from the uncertainty of the underground structure (Yagi \& Fukahata 2011; Duputel et al. 2014; Ragon et al. 2018). However, it seems none of the previous studies has not explicitly pointed out this close relationship between the two groups of studies. Because of this close relation and similarity between the approaches, it is important to point out the practical advantage of the proposed method over the naive approach, in which drawing samples from the joint PDF of $\mathbf{m}$ and $\varphi$ first and then marginalizing it with $\varphi$ after the sampling process. Figure 1 summarizes the difference between the two approaches. There are two major advantages of the proposed method over the naive approach, as discussed below.

First, the computation cost in each step of Bayesian sampling is significantly different in the two approaches. Because the relationship between the latent parameters $\varphi$ and the measurement is typically nonlinear, estimation of the joint posterior probability of $\mathbf{m}$ and $\varphi$ is typed as a nonlinear inversion problem, which requires solving the forward problem in each step of the sampling algorithm to evaluate the target PDF. In contrast, the proposed method marginalizes the likelihood function with the latent parameters and deletes them from the equation in advance of Bayesian sampling. This manner reduces the target parameter space for Bayesian sampling to only that of the model parameters, which are in a linear relationship with the measurement, allowing for evaluating the target PDF just by carrying out many matrix-vector multiplications in Equation 3 to perform Monte Carlo integration in Equation 4. Thus, if the coefficient matrices that are composed of Green's function are calculated in advance of the sampling, the sampling process itself only requires relatively simple and cheap calculations. Furthermore, because each component of the coefficient matrix can be calculated independently of each other, the use of a larger-scale computer allows for highly parallelized calculations in preparing the matrix. Sampling from a posterior distribution of such relatively high-dimensional problems as our target generally requires at least $10^{5}-10^{7}$ sequential evaluations of the target posterior PDF. Furthermore, as mentioned in Section 
2.3, MCMC, or REMC sampling, requires trial and error to find a parameter set for the proposal distributions and temperatures, with which the target PDF is properly sampled. In a problem with relatively small calculation cost for forward modeling, such as the application example in Section 4 , running the sampling algorithm based on the naive approach many times for trial and error does not cause a severe problem. However, once the calculation cost required for the forward problem is significantly large, each run of the sampling takes a significant amount of time, which makes a practical application difficult. For instance, Yamaguchi et al. (2017) calculated 1,000 sets of the coefficient matrix for a three-dimensional heterogeneous underground structure in 17 days, which corresponds to $3.6 \times 10^{5}$ executions of the forward problem, using a highly tuned computation program and 64 GPUs. It is impossible to spend the equivalent amount of time and resources to run the same amount of forward problems in carrying out one trial of sampling.

Secondly, because the model and latent parameters are usually in a strong trade-off relationship, the efficiency of Bayesian sampling is expected to be largely improved if the sampling algorithm explores only the model parameter space. The proposed method allows for sampling only from the model parameter space by marginalizing and removing the latent parameter from the PDF in advance of the sampling process. Avoiding naive sampling from both spaces of the model and latent parameters has also been an important problem in previous studies. For instance, Malinverno \& Briggs (2004) and Fukuda \& Johnson (2010) utilized the fact that the relationship between the model parameters and the measurement is linear to develop an efficient sampling algorithm from the joint PDF, separating the linear and nonlinear part of the estimation problem. Their approaches still require Bayesian sampling from the latent parameter space, which is in nonlinear relationship with the measurement. Therefore, because the latent parameters characterize the forward model prediction, it is still necessary to solve the forward problem for each step of the iterative sampling algorithm. For the problems in which the computation cost for the forward problem is moderate, their approach should be effective as well.

Moreover, the proposed method estimates the posterior PDF of the latent parameters in the form of importance sampling, as in Equation 15. The importance sampling allows for accurate approximation of the PDF with a fewer number of samples than the ordinary sampling methods, 


\section{Non-peer reviewed manuscript submitted to Geophys. J. Int. by R. Agata}

which is adopted in the naive approach. Additionally, it enables us to preserve the particles that describe the tails of the PDF with a smaller sample size. The drawback of the proposed method is that the information of associations between $\mathbf{m}$ and $\varphi$ cannot be obtained, while we expect that it is practically difficult to handle this information to make a geophysically meaningful discussion.

\section{APPLICATION TO A TOY MODEL PROBLEM OF GEODETIC SLIP ESTIMATION}

In this section, a simple 2D synthetic test of a geodetic inversion problem that includes a significant amount of prediction errors is presented. Through this example, we demonstrate the capability of the proposed method in estimating fault slip distribution in a situation under which information about the underground structure is uncertain.

\subsection{Problem setting}

We consider an estimation problem of slip deficit rate (SDR) on a dip-slip fault embedded in a 2D elastic homogeneous half-space, which models a thrust fault. The fault geometry is shown in Figure 2 (a): the fault width is considered to be $100 \mathrm{~km}$, and the true dip angle of the fault is considered to be $15^{\circ}$. The true SDR distribution shown in Figure 2 (b) is inputted in the fault plane. The fault plane is divided into ten small faults of $10 \mathrm{~km}$ width, each of which has a constant slip. The virtual observation points are distributed on the surface of the half-space, which mimics geodetic observation of the interseismic surface displacement rate (DR). We add artificial Gaussian noise to the calculated displacements, for which the standard deviations are $1.0 \times 10^{-4} \mathrm{~m} / \mathrm{yr}$ for the horizontal component and $1.0 \times 10^{-3} \mathrm{~m} / \mathrm{yr}$ for the vertical component. We also consider an additional case with larger observation errors, in which the standard deviation for the horizontal component is $1.0 \times 10^{-3} \mathrm{~m} / \mathrm{yr}$. We call the dataset in the former case Dataset 1 and that in the latter Dataset 2. Dataset 2 is used only in the problems Section 4.2.2. Figure 2 (c) illustrates the two synthetic datasets in the observation points. The covariance matrix $\mathbf{E}$ for the observation errors is constructed based on these values of standard deviations.

In estimating the posterior PDF of the SDR distribution, we assume a scenario wherein only uncertain and inaccurate information of the dip angle is available. That is, $\theta$ is assumed to be a 
variable following the Gaussian distribution with the mean $18^{\circ}$ and the standard deviation $3^{\circ}$ (i.e., $\mathcal{N}\left(18^{\circ},\left(3^{\circ}\right)^{2}\right)$ ), which corresponds to the prior PDF of $\boldsymbol{\varphi}$ or $P(\varphi)$ (i.e., $\boldsymbol{\varphi}=\varphi=\theta$ ). The uncertainty and inaccuracy regarding $\varphi$ is the source of prediction errors in this estimation problem. From this PDF, we draw 1,000 random samples of the dip angle $\left\{\theta^{(1)} \ldots \theta^{(1,000)}\right\}$ and calculate the ensemble of the coefficient matrices $\left\{\mathbf{G}\left(\varphi^{(1)}\right) \ldots \mathbf{G}\left(\varphi^{(1,000)}\right)\right\}$ based on the samples. We use $U(-0.15 \mathrm{~m} / \mathrm{yr}, 0.01 \mathrm{~m} / \mathrm{yr})$ for SDR in each small fault as the prior PDF $P(\mathbf{m})$, where $U(a, b)$ denotes a uniform probability distribution from $a$ to $b$ : we do not impose a strong assumption of the characteristic of spatial slip/SDR distribution such as regularization of a smoothness constraint (e.g. Yabuki \& Matsu'ura (1992)) or sparsity promoting L1-norm (e.g. Nakata et al. (2017)). We calculate both the synthetic data and the coefficient matrices based on the analytical expression of elastic deformation due to a 2D dipping fault embedded in an elastic half-space described in Segall (2010). Based on the synthetic data, the covariance matrix for the observation errors, and the ensemble of the coefficient matrices, we perform the REMC sampling of the posterior PDF using the parameters. Table 1 presents the parameters for REMC sampling that are chosen by trial and error.

\subsection{Results}

Figure 3 shows the sampled posterior PDF. In the left panel, smaller variances in the shallower part of the fault and larger ones in the deeper part are seen in the estimated PDF of SDR. Despite that the prior information on the dip angle is inaccurate and uncertain, the true value of SDR falls within the range of the estimated PDFs, which indicates that the stochastic property of the parameters estimated by the proposed method is mathematically consistent with the synthetic data. The right panel shows the weighted histogram consisting of 1,000 samples that approximate the posterior PDF of $\theta .94 .7 \%$ of the samples with weight fall within $\theta=14.85^{\circ}$ and $15.15^{\circ}$. It follows that the value of the dip angle is correctly estimated with small uncertainty. Figure 4 shows the posterior predicted distribution based on the samples from the posterior PDF. Plot of the posterior 
predicted distribution is produced based on the equation below:

$$
\begin{aligned}
P(\mathbf{d} \mid \mathbf{D}) & =\int P(\mathbf{d} \mid \mathbf{m}, \boldsymbol{\varphi}) P(\mathbf{m}, \boldsymbol{\varphi} \mid \mathbf{D}) d \mathbf{m} d \boldsymbol{\varphi} \\
& =\int P(\mathbf{d} \mid \mathbf{m}, \boldsymbol{\varphi}) P(\mathbf{D} \mid \mathbf{m}, \boldsymbol{\varphi}) P_{\text {new }}(\mathbf{m}) P(\boldsymbol{\varphi}) d \mathbf{m} d \boldsymbol{\varphi} \\
& \simeq \frac{1}{M} \sum_{m=1}^{M} \int P\left(\mathbf{d} \mid \mathbf{m}^{(m)}, \boldsymbol{\varphi}\right) P\left(\mathbf{D} \mid \mathbf{m}^{(m)}, \boldsymbol{\varphi}\right) P(\boldsymbol{\varphi}) d \boldsymbol{\varphi} \\
& \simeq \frac{1}{N M} \sum_{n=1}^{N} \sum_{m=1}^{M} P\left(\mathbf{d} \mid \mathbf{m}^{(m)}, \boldsymbol{\varphi}^{(n)}\right) P\left(\mathbf{D} \mid \mathbf{m}^{(m)}, \boldsymbol{\varphi}^{(n)}\right),
\end{aligned}
$$

where $\mathbf{D}, P(\mathbf{m} \mid \mathbf{D})$ and $P(\boldsymbol{\varphi} \mid \mathbf{D})$ are the observation data used for estimating the posterior PDF of SDR, posterior PDF of the model and latent parameters obtained using $\mathbf{d}=\mathbf{D}$, respectively. $\mathbf{m}^{(m)} \mathrm{S}$ and $\varphi^{(n)}$ s are regarded herein as the samples drawn from $P(\mathbf{m} \mid \mathbf{D})$ and $P(\varphi \mid \mathbf{D})$, respectively. The ordinary Monte Carlo integration for $\mathbf{m}$ is applied to the approximation from Equation 18 to 19 . The approximation from Equation 19 to 20 is based on the Monte Carlo integration for $\varphi$ with importance sampling defined in Equation 15. As the figure shows, the synthetic observation data fall within the range of the predicted distribution as well. We consider this problem setting as the base case and perform comparisons with several different settings, aiming for detailed discussions.

\subsubsection{Robustness of the estimation result versus different assumptions on the mean value of the underground structure parameters -Comparison with a conventional approach based on implicit consideration of prediction errors-}

We estimate the posterior PDF of SDR based on a different assumption of the mean value of the dip angle and examine the difference in the estimation result. Figure 5 (a) shows the results in the case where $\theta$ follows $\mathcal{N}\left(12^{\circ},\left(3^{\circ}\right)^{2}\right)$ and Dataset 1 is used. The estimated PDF appears almost identical to that obtained in the base case shown in Figure 3 (a). It follows that accurate consideration of the effect of the prediction errors on the slip estimation leads to obtaining similar estimation results despite the difference in the assumption on the mean value of the prior PDF for the dip angle.

We additionally compare the estimation results obtained using the proposed method and that based on a conventional approach that does not explicitly distinguish the prediction errors from the observation errors. For instance, such an approach is taken in combination with smoothing con- 
straints in widely applied inversion analysis methods of slip distribution (e.g., Yabuki \& Matsu'ura (1992)). In these methods, the use of a smoothing constraint introduces a penalty due to the spatial roughness of a slip distribution to the evaluation function. To perform an estimation with a prior constraint of the smoothness of the slip distribution, we construct a Bayesian model with unknown hyperparameters and draw sampling from the posterior PDF regarding the SDR distribution. This method we implement herein is adopted from Yabuki \& Matsu'ura (1992), which determined the hyperparameters using an information criterion instead of performing Bayesian sampling (see Appendix A for details).

Figure 5 (b) shows the estimated PDF using the conventional approach with $\theta=18^{\circ}$ (the standard deviation of $\theta$ is not discussed because the uncertainty of the parameter on the underground structure cannot be introduced in the conventional approach). The true values in some of the small faults are quite distant from the main part of the histograms, particularly in the shallowest and the deepest edge of the fault. Figure 6 shows that the predicted distribution well-explains the horizontal component of the data, which is associated with smaller errors. However, the model poorly explains the vertical component, i.e., overfitting the data in the horizontal component occurs because important characteristics of the noise, such as covariance components in the prediction errors (e.g., Yagi \& Fukahata $(2008,2011)$ ), are missing in the formulation. This situation results in the estimation of the posterior PDF that is statistically inconsistent with the true model parameters. Figure 5 (c) shows the same estimation with a different assumption for the dip angle, $\theta=12^{\circ}$. The histograms appear to be quite different from the true values and those obtained with $\theta=18^{\circ}$. In the cases where a significant amount of the prediction errors is included in the prediction model, a conventional approach that does not distinguish the prediction errors from observation errors can produce estimation results that are highly dependent on the model assumptions, which is contrary to the results obtained using the proposed method. These findings are consistent with the arguments made by Minson et al. (2013) and Duputel et al. (2014) on the discrepancy in the published fault slip models for the same earthquake, which we mention in Section 1. 


\subsubsection{Variation of the solution versus different assumptions on the uncertainty of the underground structure}

The results in Section 4.2.1 suggest that the proposed method can produce estimation results that are less dependent on an assumption on the mean value of the underground structure parameter as long as the variance is set correctly. Next, we examine the importance of the variance, or uncertainty in a more general context, in the estimation. To show the impact of changing the variance on estimation results more clearly, we use Dataset 2, in which the accuracy of synthetic DR data for the horizontal component is one-order lower than Dataset 1 . We consider three cases where the dip angle follows $\mathcal{N}\left(18^{\circ},\left(3^{\circ}\right)^{2}\right), \mathcal{N}\left(18^{\circ},\left(6^{\circ}\right)^{2}\right)$ and $\mathcal{N}\left(18^{\circ},\left(0.85^{\circ}\right)^{2}\right)$, which we call Case 1 , 2 , and 3 , respectively.

Figure 7 (a) shows the results in Case 1 , where $\theta$ follows $\mathcal{N}\left(18^{\circ},\left(3^{\circ}\right)^{2}\right)$. Because the data are less accurate, the uncertainty in the estimation results of both the SDR and the dip angle becomes larger than that in the base case. We additionally observe that the shape of the histogram is slightly biased from the true value for the mean value of the prior PDF, $18^{\circ}$. Because of the large uncertainty that we assume for the observation errors, more emphasis is put on the prior dip angle information than in the base case. In the posterior prediction distribution (Figure 8), gentler function shapes than those with Dataset 1 are drawn in the horizontal components, which also reflects the larger variance for the observation errors.

The estimated PDFs in Case 2 (Figure 7 (b)), where $\theta$ follows $\mathcal{N}\left(18^{\circ},\left(6^{\circ}\right)^{2}\right)$, have also basically the same characteristics as the result obtained in Case 1. The histogram for the estimated dip angle looks quite like that of Case 1 . However, the histogram for Case 2 is less biased for the mean value of the dip angle. Figure 9 shows closeup views of the samples of the posterior PDF for SDR and the posterior prediction PDF as examples. The drawn curves for Case 2 look almost identical to those for Case 1. After additional trials, we found that the basic characteristics stay the same even when a larger $\sigma$ is adopted for the PDF of the dip angle. This finding can be understood as follows. As the variance of the dip angle increases, the true value $\theta=15^{\circ}$ for the assumed PDF of the dip angle increases as well. For example, the true value $\theta=15^{\circ}$ corresponds to $1 / 2-\sigma$ value for $\mathcal{N}\left(18^{\circ},\left(6^{\circ}\right)^{2}\right)$, while corresponding to $1-\sigma$ for $\mathcal{N}\left(18^{\circ},\left(3^{\circ}\right)^{2}\right)$ : The true value $\theta=15^{\circ}$ 
becomes a more likely realization as the variance becomes larger in the assumed PDF. In another viewpoint, when large uncertainty on the prior information of the underground structure parameter is assumed, more emphasis is put on the observation data. Thus, the results almost exclusively reflect the information obtained from the observation data.

In Case 3, we estimated the posterior PDF of SDR assuming that $\theta$ follows $\mathcal{N}\left(18^{\circ},\left(0.85^{\circ}\right)^{2}\right)$, in which the true value $\theta=15^{\circ}$ corresponds to approximately 3.5- $\sigma$ (Figure 7 (c)): For this PDF, the true dip angle corresponds to an unlikely realization. In the right panel, we observe that the peak of the histogram is located near the bin of $16^{\circ}$, from which we see a significant impact from the mean value in the prior PDF. It follows that larger weight is put on the prior information of the dip angle in this case because of its relatively small uncertainty. The estimated SDR distributions in the left panel looks similar to those in Case 1 and 2. However, the closeup views of the samples of the posterior PDF for SDR and the posterior prediction PDF (Figure 9) show that there are small but significant differences between the result in Case 3 and those in the two other cases in some of the fault patches and the observation points. Notably, the three cases estimate similar function shapes for a fault patch located at a deeper portion of the fault plane (Figure 9 (b)) probably because the surface DR response to fault slip in a deeper portion is less sensitive to the change of the dip angles. Moreover, the estimates of Case 1 and 2 are not always closer to the true values than those of Case 3 (e.g., Figure 9 (c)) because of the randomness of the artificial noise included in the synthetic data.

In summary, the uncertainty of the underground structure has an impact on the estimation results by determining the balance between the observation data and the prior information on the underground structure. In our problem setting, as the variance of the dip angle increases, more emphasis is put on the information in the data. As a result, the estimation result does not strongly depend on the variance of the dip angle if the variance changes in a range of values that are large to some extent. As the variance of the dip angle decreases, more emphasis is put on the prior information. In such a situation, if the mean value of the prior PDF for the dip angle is significantly different from the true one, the estimation results of the SDR, the dip angle, and the posterior prediction of DR is less consistent with the true values. Such a tendency for the impact 
of the uncertainty is easy to understand herein because our toy problem exclusively adopts one parameter for the underground structure, and the data is sufficiently sensitive to changes in the dip angle. More complicated situations expected in real-world problems will be discussed in Section 5 .

\subsubsection{Non-Gaussianity of the prediction errors}

One of the advantages of the proposed method over the previous studies is that the assumption of the Gaussian distribution on the prediction errors is not necessary. We plot an example set of prediction errors in Figure 10 for the base case. Because the prediction errors depends on $\mathbf{m}$, we choose $\overline{\mathbf{m}}$ as a representative case and adopt $P\left(\mathbf{d}_{\text {pred }} \mid \overline{\mathbf{m}}\right)$ for plotting, where $\overline{\mathbf{m}}=\frac{1}{M} \sum_{m=1}^{M} \mathbf{m}^{(m)}$ is the mean of the samples from the posterior PDF. We observe shapes for the distribution that are significantly different from the Gaussian distribution in some observation points. The underlined numbers in the figure are the skewness, which is a statistic that can be used as a metric of the discrepancy of the target PDF from the Gaussian distribution of the estimated prediction errors at each observation point. A widely known rule of thumb for interpreting the skewness (Bulmer 1979) is:

- A distribution with a skewness less than -1 or greater than +1 is highly skewed.

- A distribution with a skewness between -1 and -0.5 or between +0.5 and +1 is moderately skewed.

- A distribution with a skewness between -0.5 and +0.5 is approximately symmetric.

The estimated PDF in more than one-third of the observation points is considered to be a highly skewed distribution, for which the Gaussian approximation might not be very appropriate. These findings indicate that the assumption of the Gaussian distribution of the prediction errors may sometimes introduce some bias to the estimation of the posterior PDF.

\subsubsection{Ensemble size to model Green's function uncertainty}

In most analyses presented so far above, we used 1,000 samples of the dip angle drawn from a PDF that represents its stochastic property. We examined whether the number of samples is enough for 
estimating the posterior PDF. Figure 11 (a)(b) shows the estimation result using 2,000 samples in the same setting as that of the base case. Little change from the result obtained using 1,000 samples is observed in the estimated PDF for the slip parameters. The posterior PDF for the dip angle estimated using 2,000 samples shows good agreement with the result in the base case with 1,000 cases in the sense that most of the samples with weight fall within $\theta=14.85^{\circ}$ and $15.15^{\circ}$. However, the case with 2,000 samples has a taller peak in the central bin. The histogram for the posterior PDF of the latent parameter appears to be more sensitive to the ensemble size because the number of samples of the latent parameter is identical to the number of items to draw the histogram. These findings indicate that 1,000 is enough ensemble size for estimating the posterior PDFs, but a detailed investigation of that for the latent parameter may require a larger ensemble size. Such a feature is quite evident in the setting of Case 3 in Section 4.2.2, which is attributed to a small variance of the assumed PDF for the dip angle. Figure 11 (c)(d) shows the results obtained using 1,000 samples of the dip angle for the same setting as Case 3. The histogram for the posterior PDF of the dip angle is unnaturally truncated in the boundary of the bins between $15^{\circ}$ and $15.5^{\circ}$, although the result for SDR looks quite consistent with other results. This result is understandable because the true value $\theta=15^{\circ}$ corresponds to 3.5- $\sigma$ in this distribution, which means that the probability that a sample of $\theta$ smaller than $15^{\circ}$ is drawn is around $2.3 \times 10^{-4} .1,000$ samples are not enough to approximate the shape of the PDF around $15^{\circ}$. The result presented in Section 4.2.2 uses 10,000 samples to show a converged solution. In such a case, the assumed PDF for the latent parameters should be revised rather than further increase the ensemble size to obtain a converged solution (see Section 5.4 for a detailed discussion). From this result, we conclude that the ensemble size that is enough to approximate the posterior PDF of the model parameters depends on the assumed PDF for the latent parameters.

The number of samples is 500,000 in all the cases presented in this study, which is a sufficiently large number to obtain a converged solution. This study only presents an example using one latent parameter. The case with multiple latent parameters will be discussed in Section 5.4. 


\section{Non-peer reviewed manuscript submitted to Geophys. J. Int. by R. Agata}

\section{DISCUSSION OF APPLICATIONS TO REAL-WORLD PROBLEMS}

The application example of the proposed method to a simple problem presented in the previous section demonstrates the basic aspects of the method. Based on these findings, we discuss the application of the proposed method to real-world problems in this section.

\subsection{Regularization-free slip inversion}

In the previous section, we compared the estimation results obtained using the proposed method and a method based on the regularization of smoothing constraints. With an aiming to discuss the results in terms of real-world applications, we first summarize the role of the regularization based on prior constraints, including that based on the smoothing constraint, in a typical slip inversion method. Regularization based on prior constraints is known to help inversion analyses in two aspects:

(i) the inverse problems in the real world are often ill-posed (in majority of such cases, nonuniqueness of the solution is not satisfied). In the worst case, the coefficient matrix is not numerically invertible. The introduction of regularization enables us to solve such inverse problems by eliminating tiny singular values of the coefficient matrix.

(ii) Even if the problem is numerically solvable, the estimation result may overfit the noise originating from the observation and model prediction errors due to the ill-conditioned nature of the problem. By balancing the information from the data and the penalty introduced by the regularization based on the physical or empirical prior knowledge, overfitting the noise is reduced. However, as Yagi \& Fukahata (2008) and Yagi \& Fukahata (2011) point out, even if a proper regularization is applied, missing important noise characteristics in the inversion scheme, such as covariance components in the prediction errors, possibly still induces overfitting and distorts the estimated slip distribution. These issues may be the reasons for the significant discrepancy of available fault slip models for the same earthquake, with which it is not easy to draw a meaningful conclusion about the source process (Minson et al. 2013; Duputel et al. 2014). Furthermore, it is generally difficult to find a regularization method that has strong scientific evidence for the slip 
inversion. However, the choice of the regularization method often largely changes the estimation result of slip distribution. For example, the introduction of a type of sparsity-promoting constraint to an estimation of slip distribution in long-term slow slip events in the Nankai Trough region in south-west Japan resulted in significantly different up- and down-dip limits for slip distribution from those obtained using smoothness constraints (Nakata et al. 2017).

The use of Bayesian inference based on mathematically rigorous treatment of the observation and prediction errors, such as presented in this study, can be an alternative solution to ill-posedness and overfitting in the slip inverse problems: Ill-posedness of the inverse problem does not become severe in the framework of Bayesian inference, because the computation of matrix inverse is not required: The non-uniqueness of the solution results in a nearly uniform posterior PDF of the target parameter. Overfitting due to the ill-conditioned system can be avoided by accurately handling the stochastic properties of the errors in the target posterior PDF. Previous studies such as Duputel et al. (2014), Ragon et al. (2018), and Gombert et al. (2018) performed slip inferences without regularization based on such an idea. However, it should be noted that an implicit smoothing constraint due to the use of constant or linear basis functions for parametrizing the slip distribution is still introduced in their method. We expect that the proposed method has the potential to be a powerful tool to perform regularization-free slip inversions. The analysis in Section 4 indicates that the proposed method can resolve the issue of discrepant source models originating from different assumptions in the underground structure, in contrast to the results obtained using the method of the ordinary smoothing constraint presented in Section 4.2.1. Notably, we did not discuss the resolution power in the estimations presented in Section 4. We applied only single patterns of fault discretization, based on which synthetic data are also calculated. Considering the issue on the resolution power of estimation when the proposed method is used as a regularization-free slip estimation method (Minson et al. 2013; Duputel et al. 2014) needs further studies in the future.

\subsection{PDF for the latent parameters and the ensemble size}

In the application of the proposed method for earthquake source estimations using geodetic or seismic waveform data, we first need to set an underground structure model associated with its 


\section{Non-peer reviewed manuscript submitted to Geophys. J. Int. by R. Agata}

uncertainty information. Because most of the available databases on the seismic velocity structure and fault geometry (e.g., Koketsu et al. (2012); Laske et al. (2013)) are not associated with the estimation errors, we usually need to set PDFs that describe the stochastic parameters by ourselves. A possible practice is to compare multiple different databases and information for the target underground structure and assume values of the mean and variance of Gaussian distribution for each parameter. An ideal situation is that the seismic velocity structure and the fault geometry associated with their uncertainty information are provided as a database based on seismic exploration, seismicity analysis, and other estimation methods. If the information is prepared in the form of an ensemble of the possible underground structure models, the proposed method can directly import the ensemble to calculate that of Green's function. Methods to estimate underground structures in the form of an ensemble has been studied recently (Fichtner et al. 2019; Gebraad et al. 2020).

The results shown in Section 4.2.4 indicate that unboundedness of the assumed PDF sometimes leads to a demand for an enormous ensemble size to obtain a converged posterior PDF. Based on our knowledge of nature, it is usually reasonable to consider the range of latent parameters as bounded rather than unbounded. In such a case, the use of a PDF that is bounded on one side or both, such as the Gamma distribution or the truncated normal distribution, by making use of the flexibility of the ensemble-based method in terms of choice of PDFs, may be more appropriate than using an unbounded distribution such as the Gaussian distribution. The discussion in Section 4.2.4 additionally suggests that the difficulty in obtaining a converged posterior PDF indicates an inconsistency of the assumed PDF of latent parameters for the real underground structure because such difficulty may have originated from a low likelihood of the true value for the assumed PDF.

\subsection{PDF for the latent parameters and the estimation results}

We discuss the impact of the prior information of a latent parameter on the estimation results. We first focus on the impact on the estimation results of the model and the latent parameters. As seen in Section 4.2.2, the estimation results almost directly reflects the information in the observation data if we set a large uncertainty for the latent parameter. If the uncertainty for the latent parameter is small and a significant amount of bias from the true value is included in the prior information, 
the estimation results of the model and latent parameter also include bias. The examples in Section 4.2.2 present only a small bias in the results, but it is only because of the small bias in the prior information for the latent parameter.

Further, we discuss the impact of the prior information on a latent parameter on the prediction. If the data contains sufficient information on the latent parameter, a bias in the prior information on the latent parameter results in a bias in the posterior prediction PDF, as well as in the estimation of the model and the latent parameters. On the other hand, predicting the response at a point in the space and time where the data does not exist is sometimes fundamentally important. Even if the data does not contain enough information on a latent parameter, accurate information on the latent parameter with small bias and low uncertainty can be a key factor in carrying out accurate predictions. The meaning of "prediction" herein is not limited to the calculated response based on the estimated slip distribution, but also applies to the prediction of events in the future, e.g., prediction of post-earthquake deformation based on an estimated coseismic slip model (Iinuma 2018) and dynamic rupture scenario of an earthquake based on an estimation of slip deficit rates in interseismic periods (Hok et al. 2011).

Notably, the proper parametrization of the underground structure, such as decomposition of the structure into stratified layers and choice of controlling parameters of the fault geometry, essentially requires knowledge of the structure.

\subsection{Multiple latent parameters and the ensemble size}

In applying the proposed method to real-world problems, we need to introduce at least several latent parameters to parametrize the stochastic properties of the target underground structure. In future work, we need a more general discussion about the ensemble size for such a case, because consideration of a high-dimensional parameter space may cause a severe problem in terms of computation cost due to requirement of a large ensemble size, which can be considered as a type of "the curse of dimensionality" (Bellman 1957).

Provided that we focus on the estimation of the model parameters, the practice of using the Ensemble Kalman Filter code(EnKF) is encouraging: EnKF usually uses a modest ensemble size 
(i.e., of the order of $10^{2}$ ) to approximate the stochastic property of the errors of the data vector in a typical meteorological problem. This ensemble size is used because the response in the observation space is spatially localized due to the nature of the governing equation. In the problems of seismology, this should also be the case, at least, for the slip estimations using geodetic data, because the elastic deformation, which rapidly decays with distance, is the basic physics for the problems. In the estimations using seismic waveform data, we may need a larger ensemble size than in the geodetic estimations in some problem settings, because the Green's function is less localized spatially in the wave equation. Further study may be required to overcome the potentially larger computation cost associated with the application of the proposed method to such problems.

\subsection{Updating the information on the underground structure through each event}

The proposed method can update the information on the underground structure via earthquake source estimation using seismic waveforms, geodetic data, and/or other kinds of observation data regarding earthquakes. When a new event is analyzed following an analysis of a previous event in the same region, a new underground structure model that was updated via the previous analysis can be used. From this viewpoint, hypocenter determination is one of the expected application examples of such capability of the proposed method. In hypocenter determination problems, travel times are calculated using the Ray path that is based on the assumed underground structure. Therefore, properly constructing the three-dimensional seismic velocity model has been aimed to determine the hypocenters (Husen et al. 2003; Nakano et al. 2015) accurately. The introduction of the proposed method should decrease the bias in the results of hypocenter determination due to the deterministic use of the $3 \mathrm{D}$ velocity structure and update the structure through the analysis simultaneously.

\section{CONCLUSIONS}

We developed a flexible Bayesian inference method for estimating fault slips that can accurately incorporate non-Gaussian prediction errors, considering the uncertainty of the underground structure based on ensemble modeling. The flexibility of the probability distribution shape is main- 
tained by performing the integration for evaluating the likelihood function based on the Monte Carlo approximation using the samples in the ensemble of the Green's function, instead of assuming a Gaussian distribution that allows for analytically evaluating the integration. The framework can additionally estimate the posterior PDF of the parameters of the underground structure by evaluating the likelihood of each sample in the ensemble through the Bayesian sampling.

To validate the advantage of the proposed method, we performed simple numerical experiments for estimating SDR distributions on a 2D thrust fault using synthetic data of surface displacement rates, in which the dip angle of the fault plane is the parameter used to characterize the underground structure. In a scenario wherein the information on the dip angle is uncertain and inaccurate, the proposed method succeeded in estimating posterior PDFs of SDR that are consistent with the true SDR, even for different mean values of the prior Gaussian PDF. Furthermore, this method could estimate a posterior PDF of the dip angle that has a strong peak around the true value. With equivalently inaccurate dip angles, the results obtained using a conventional approach, which does not explicitly distinguish the prediction and observation errors and introduces regularization based on smoothing constraints, included a significant amount of bias, which was not witnessed in in the experiments using the proposed method. We also applied different values of the variance of the prior PDF for the dip angle and examined the impact on the estimation results. As a result, if the variance varied in a range of values that are large to some extent, the estimation result did not strongly depend on the variance of the dip angle. In contrast, as the variance of the dip angle becomes smaller, more emphasis was put on the prior information. In such a case, when the mean value of the prior PDF of the dip angle was significantly different from the true one, the estimation results of the dip angle was significantly biased for the mean value. As a result,, the estimation of the posterior PDF of SDR and the posterior prediction of DR also included a significant amount of bias from those estimated based on a larger variance in the prior PDF of the dip angle. Moreover, the prior PDF of the dip angle that was inconsistent with the true value (i.e., the likelihood of the true value for the prior PDF is very small) required a significantly larger ensemble size to obtain convergence of the posterior PDF. The distribution shapes of the prediction errors for the representative model parameters in some observation points were quite asymmetric, with the 
absolute value of the sample skewness larger than one, for which Gaussian approximation is not usually applied. This result indicates that the flexibility of the probability distribution shape due to the application of ensemble-based modeling may contribute to the high accuracy of the analyses.

We further discussed the important points to consider in applying the proposed method to realworld problems. We suggested that the proposed method can be a candidate as a powerful tool for regularization-free slip inversions because our Bayesian sampling algorithm that accurately handles Non-Gaussian prediction errors can provide an alternative solution to the ill-posedness and ill-conditioning of the inverse problems. Based on the speculation that the spatial locality of the response due to the nature of the governing equation reduces the ensemble size that is necessary to model the uncertainty of underground structure, geodetic slip inversions using elastic deformation models, in which the response decays rapidly with distance, are expected to be a relatively easier problem to take on. On the contrary, in the problems using the seismic waveform data, such as waveform slip inversions and hypocenter determinations, simultaneous estimation of the source and the underground structure should become more interesting topic because the data consist of waves from many paths and are expected to include more information of the underground structure. In return, more computation efforts may be required to deal with a large amount of computation cost due to the large ensemble size.

\section{ACKNOWLEDGMENT}

I greatly appreciate the comments on Bayesian sampling methods by Dr. Hiromichi Nagao and Dr. Shin-ichi Ito. This research was supported by JSPS KAKENHI Grant Number JP19H04631 in Scientific Research on Innovative Areas "Science of Slow Earthquakes". Some figures were produced using GMT software (Smith \& Wessel 1990).

\section{REFERENCES}

Agata, R., 2020. Introduction of covariance components in slip inversion of geodetic data following a nonuniform spatial distribution and application to slip deficit rate estimation in the Nankai Trough subduction zone, Geophysical Journal International, 221(3), 1832-1844. 
Agata, R., Ichimura, T., Hori, T., Hirahara, K., Hashimoto, C., \& Hori, M., 2018. An adjoint-based simultaneous estimation method of the asthenosphere's viscosity and afterslip using a fast and scalable finite-element adjoint solver, Geophysical Journal International, 213(1), 461-474.

Bellman, R., 1957. Dynamic Programming, Dover Publications.

Bishop, C. M., 2006. Pattern recognition and machine learning, springer.

Bulmer, M. G., 1979. Principles of statistics, Courier Corporation.

Duputel, Z., Agram, P. S., Simons, M., Minson, S. E., \& Beck, J. L., 2014. Accounting for prediction uncertainty when inferring subsurface fault slip, Geophysical Journal International, 197(1), 464-482.

Evensen, G., 1994. Sequential data assimilation with a nonlinear quasi-geostrophic model using monte carlo methods to forecast error statistics, Journal of Geophysical Research: Oceans, 99(C5), 1014310162.

Fichtner, A., Zunino, A., \& Gebraad, L., 2019. Hamiltonian Monte Carlo solution of tomographic inverse problems, Geophysical Journal International, 216(2), 1344-1363.

Fukahata, Y. \& Wright, T. J., 2008. A non-linear geodetic data inversion using ABIC for slip distribution on a fault with an unknown dip angle, Geophysical Journal International, 173(2), 353-364.

Fukuda, J. \& Johnson, K. M., 2010. Mixed linear-non-linear inversion of crustal deformation data: Bayesian inference of model, weighting and regularization parameters, Geophysical Journal International, 181(3), 1441-1458.

Gebraad, L., Boehm, C., \& Fichtner, A., 2020. Bayesian Elastic Full-Waveform Inversion Using Hamiltonian Monte Carlo, Journal of Geophysical Research: Solid Earth, 125(3), e2019JB018428, e2019JB018428 10.1029/2019JB018428.

Geyer, C. J., 1991. Markov chain Monte Carlo maximum likelihood.

Gombert, B., Duputel, Z., Jolivet, R., Simons, M., Jiang, J., Liang, C., Fielding, E. J., \& Rivera, L., 2018. Strain budget of the ecuador-colombia subduction zone: A stochastic view, Earth and Planetary Science Letters, 498, 288-299.

Gordon, N. J., Salmond, D. J., \& Smith, A. F., 1993. Novel approach to nonlinear/non-Gaussian Bayesian state estimation, in IEE proceedings F (radar and signal processing), vol. 140, pp. 107-113, IET.

Hallo, M. \& Gallovič, F., 2016. Fast and cheap approximation of green function uncertainty for waveformbased earthquake source inversions, Geophysical Journal International, 207(2), 1012-1029.

Hok, S., Fukuyama, E., \& Hashimoto, C., 2011. Dynamic rupture scenarios of anticipated nankai-tonankai earthquakes, southwest japan, Journal of Geophysical Research: Solid Earth, 116(B12).

Husen, S., Kissling, E., Deichmann, N., Wiemer, S., Giardini, D., \& Baer, M., 2003. Probabilistic earthquake location in complex three-dimensional velocity models: Application to Switzerland, Journal of Geophysical Research: Solid Earth, 108(B2).

Iinuma, T., 2018. Postseismic uplift along the pacific coast of Tohoku and Kanto districts associated with 
the 2011 off the Pacific Coast of Tohoku Earthquake, Journal of Disaster Research, 13(3), 496-502.

Kano, M., Nagao, H., Ishikawa, D., Ito, S.-i., Sakai, S., Nakagawa, S., Hori, M., \& Hirata, N., 2017. Seismic wavefield imaging based on the replica exchange monte carlo method, Geophysical Journal International, 208(1), 529-545.

Kitagawa, G., 1993. A Monte Carlo filtering and smoothing method for non-Gaussian nonlinear state spacemodels, Technical report, Institute of Statistical Mathematics Research Memorandum, (462).

Kitagawa, G., 1996. Monte Carlo filter and smoother for non-Gaussian nonlinear state space models, Journal of computational and graphical statistics, 5(1), 1-25.

Koketsu, K., Miyake, H., \& Suzuki, H., 2012. Japan integrated velocity structure model version 1, Proceedings of the 15th World Conference on Earthquake Engineering, (1773).

Kubo, H., Asano, K., Iwata, T., \& Aoi, S., 2016. Development of fully Bayesian multiple-time-window source inversion, Geophysical Journal International, 204(3), 1601-1619.

Laske, G., Masters, G., Ma, Z., \& Pasyanos, M., 2013. Update on CRUST1.0 - A 1-degree global model of Earth's crust, EGU General Assembly Vienna, Austria, 15, 2658.

Malinverno, A. \& Briggs, V. A., 2004. Expanded uncertainty quantification in inverse problems: Hierarchical Bayes and empirical Bayes, Geophysics, 69(4), 1005-1016.

Metropolis, N., Rosenbluth, A. W., Rosenbluth, M. N., Teller, A. H., \& Teller, E., 1953. Equation of state calculations by fast computing machines, The journal of chemical physics, 21(6), 1087-1092.

Minson, S., Simons, M., \& Beck, J., 2013. Bayesian inversion for finite fault earthquake source models I-Theory and algorithm, Geophysical Journal International, 194(3), 1701-1726.

Nakano, M., Nakamura, T., \& Kaneda, Y., 2015. Hypocenters in the Nankai Trough Determined by Using Data from Both Ocean-Bottom and Land Seismic Networks and a 3D Velocity Structure Model: Implications for Seismotectonic Activity, Bulletin of the Seismological Society of America, 105(3), 15941605 .

Nakata, R., Hino, H., Kuwatani, T., Yoshioka, S., Okada, M., \& Hori, T., 2017. Discontinuous boundaries of slow slip events beneath the Bungo Channel, southwest Japan, Scientific reports, 7(1), 6129.

Ragon, T., Sladen, A., \& Simons, M., 2018. Accounting for uncertain fault geometry in earthquake source inversions-I: theory and simplified application, Geophysical Journal International, 214(2), 1174-1190.

Rosenblatt, M., 1956. Remarks on some nonparametric estimates of a density function, The Annals of Mathematical Statistics, 27, 832-837.

Segall, P., 2010. Earthquake and volcano deformation, Princeton University Press.

Smith, W. \& Wessel, P., 1990. Gridding with continuous curvature splines in tension, Geophysics, 55(3), 293-305.

Swendsen, R. H. \& Wang, J.-S., 1986. Replica Monte Carlo simulation of spin-glasses, Physical review letters, 57(21), 2607. 
Yabuki, T. \& Matsu'ura, M., 1992. Geodetic data inversion using a Bayesian information criterion for spatial distribution of fault slip, Geophysical Journal International, 109(2), 363-375.

Yagi, Y. \& Fukahata, Y., 2008. Importance of covariance components in inversion analyses of densely sampled observed data: an application to waveform data inversion for seismic source processes, Geophysical Journal International, 175(1), 215-221.

Yagi, Y. \& Fukahata, Y., 2011. Introduction of uncertainty of green's function into waveform inversion for seismic source processes, Geophysical Journal International, 186(2), 711-720.

Yamaguchi, T., Ichimura, T., Yagi, Y., Agata, R., Hori, T., \& Hori, M., 2017. Fast crustal deformation computing method for multiple computations accelerated by a graphics processing unit cluster, Geophysical Journal International, 210(2), 787-800. 
32 Non-peer reviewed manuscript submitted to Geophys. J. Int. by R. Agata

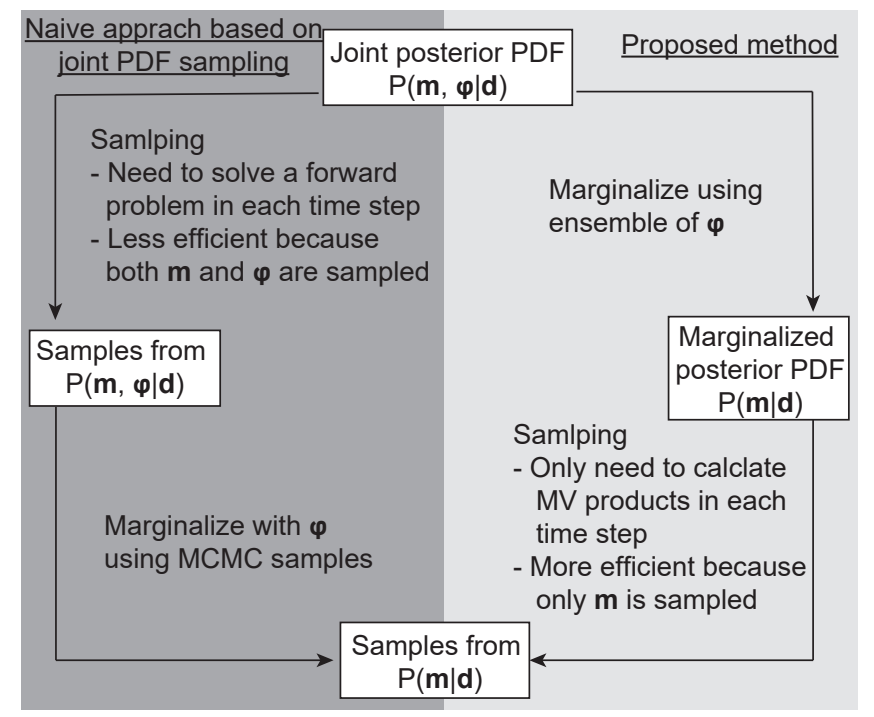

Figure 1. Summary of the comparison the proposed method and a naive approach based on Bayesian sampling from the joint PDF in performing Bayesian sampling of $P(\mathbf{m} \mid \mathbf{d})$.

Table 1. Parameters for the REMC samlping performed for the base case.

\begin{tabular}{cccccc}
$M_{\text {iteration }}$ & $M_{\text {burnin }}$ & $M_{\text {exchange }}$ & $L$ & $T_{2}$ & $\beta_{k}$ \\
\hline 500,000 & 10,000 & 10 & 20 & 2 & $3.0 \times 10^{-4} \mathrm{~m} / \mathrm{yr}$
\end{tabular}




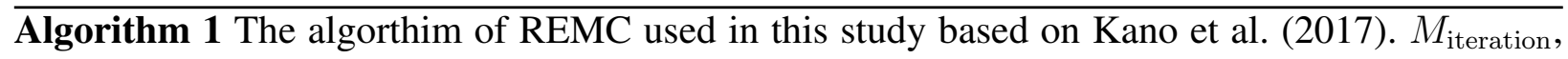
$M_{\text {burnin }}, M_{\text {exchange }}, L, \mathbf{m}_{l}^{(m)}, T_{l}, K$, and $\beta_{k}$ are the number of iteration, that of the samples for burn-in period, that of the iterations every of which the chains are exchanged, that of the chains, the model parameter in the $l$-th chain at the $m$-th step, the temperature in the $l$-th chain, the number of the model parameters, and the standard deviation of the $k$-th model parameter. $U(a, b)$ denotes a uniform probability distribution from $a$ to $b \cdot \operatorname{Mod}(\cdot, \cdot)$ denotes the Modulo operator.
\end{abstract}

Input: $M_{\text {iteration }}, M_{\text {burnin }}, M_{\text {exchange }}, L, \mathbf{m}_{l}^{(0)}, T_{l}(l=1, \ldots, L), K, \beta_{k}(k=1, \ldots, K)$

Output: $\mathbf{m}_{1}^{(m)}\left(m=M_{\text {burnin }}, \ldots, M_{\text {iteration }}\right)$ as the samples drawn from the target PDF

$P_{l}(\mathbf{m})=P(\mathbf{m} \mid \mathbf{d})^{1 / T_{l}}(l=1, \ldots, L)$ (Define a family of PDFs)

for $m=0$ to $M-1$ do

$$
\begin{aligned}
& \mathbf{m}_{l}^{*} \Leftarrow \mathbf{m}_{l}^{(m)}+\boldsymbol{\pi}_{l}, \boldsymbol{\pi}_{l} \sim N\left(\mathbf{0}, \operatorname{diag}\left(\beta_{k}^{2}\right)\right) \\
& P_{l}^{\text {accept }} \Leftarrow \min \left[1, \frac{P_{l}\left(\mathbf{m}_{l}^{*}\right)}{P_{l}\left(\mathbf{m}_{l}^{(m)}\right)}\right] \\
& u_{l} \Leftarrow(\text { a random sample from } U(0,1)) \\
& \text { if } u_{l} \leq P_{l}^{\text {accept }} \text { then } \\
& \quad \mathbf{m}_{l}^{(m+1)} \Leftarrow \mathbf{m}_{l}^{*}
\end{aligned}
$$

else

$$
\mathbf{m}_{l}^{(m+1)} \Leftarrow \mathbf{m}_{l}^{(m)}
$$

\title{
end if
}

if $\operatorname{Mod}\left(m, M_{\text {exchange }}\right)=0$ then

$$
u \Leftarrow(\text { a random sample from } U(0,1))
$$

Select randomly a pair of consecutive $l$-th and $l+1$-th chains

$$
P_{\text {accept }} \Leftarrow \min \left[1, \frac{P_{l}\left(\mathbf{m}_{l+1}^{(m)}\right) P_{l+1}\left(\mathbf{m}_{l}^{(m)}\right)}{P_{l}\left(\mathbf{m}_{l}^{(m)}\right) P_{l+1}\left(\mathbf{m}_{l+1}^{(m)}\right)}\right]
$$

if $u \leq P_{\text {accept }}$ then

Work in parallel for $l=1, \ldots, L$

$$
\begin{aligned}
\mathbf{m}_{l+1}^{(m)} & \Leftarrow \mathbf{m}_{l}^{(m)} \\
\mathbf{m}_{l}^{(m)} & \Leftarrow \mathbf{m}_{l+1}^{(m)}
\end{aligned}
$$

end if

end if 


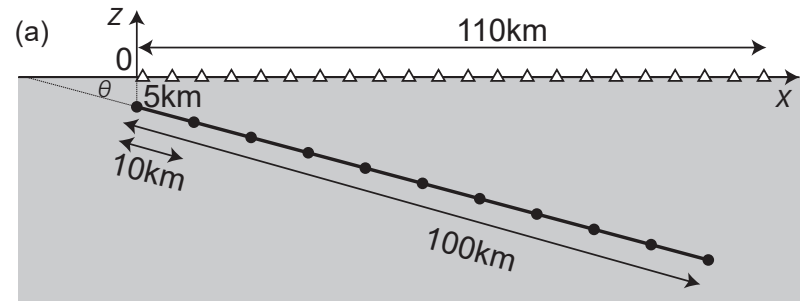

(c) $\circ$ Dataset $1 \times$ Dataset 2

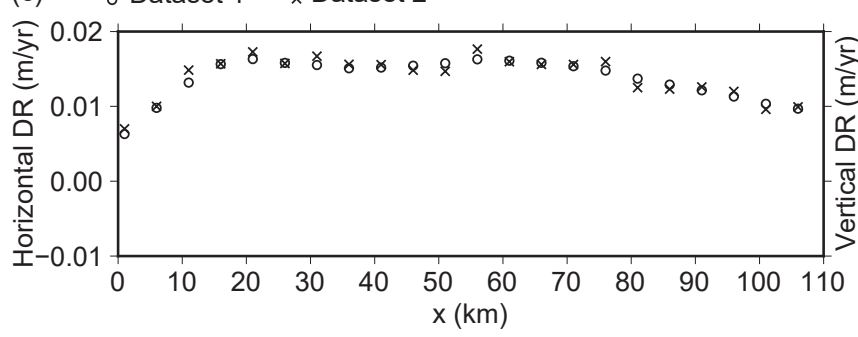

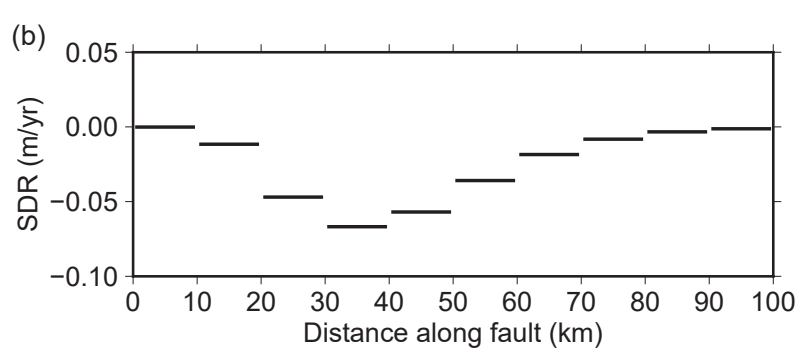

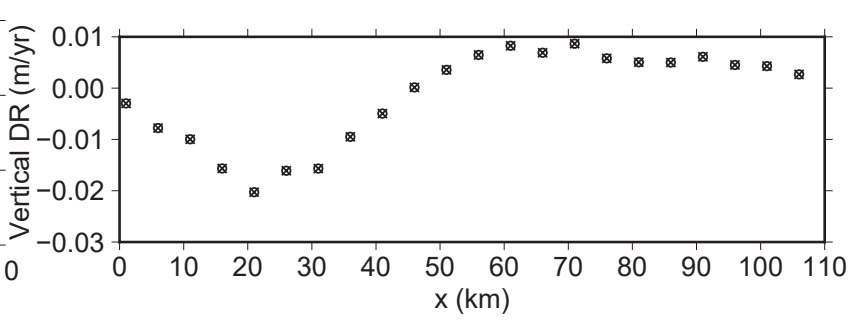

Figure 2. Problem setting of the synthetic tests. (a) Fault geometry. The black dots and white triangles denote the boundaries of the small faults and the observation point, respectively. The entire fault width and small fault width are taken to be $100 \mathrm{~km}$ and $10 \mathrm{~km}$, respectively. The true dip angle of the fault $\theta=15^{\circ}$. (b) The true SDR distribution inputted on the fault plane. A constant value is taken in each small fault. (c) The synthetic data of displacement rate (DR) in Dataset 1 and 2 calculated based on the true SDR distribution and the fault geometry. Gaussian noises are added to the calculated DR. The data in the two datasets in the vertical component overlaps because their difference is only in the amount of the observation errors in the horizontal component.
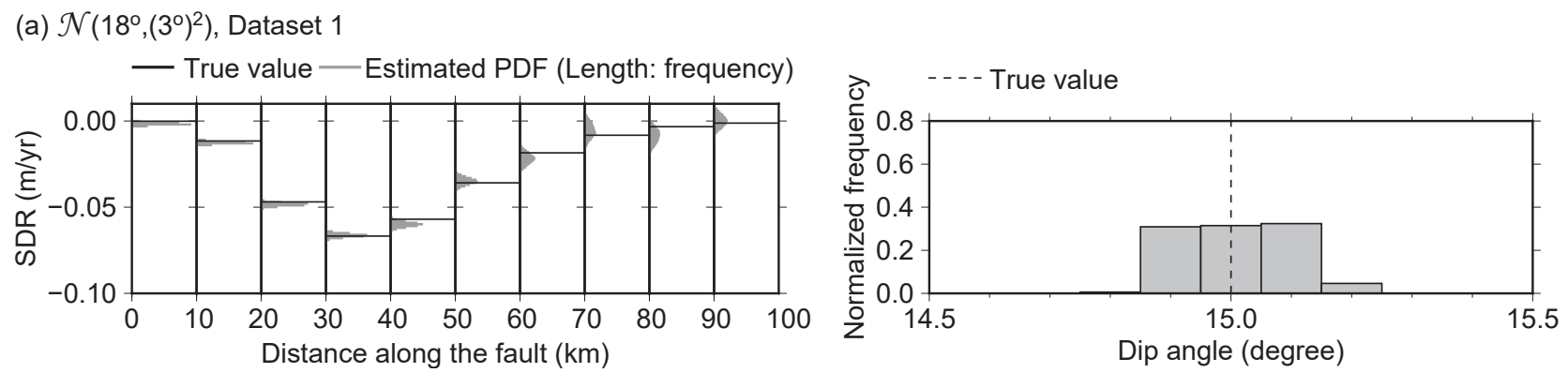

Figure 3. The estimation result in the base case, using Dataset 1 and assuming that $\theta$ follows $\mathcal{N}\left(18^{\circ},\left(3^{\circ}\right)^{2}\right)$. The left panel shows the estimation result of the posterior PDF of SDR and the dip angle. The right panel shows the posterior predicted PDF of horizontal and vertical displacement rate (DR). 
$\mathcal{N}\left(18^{\circ},\left(3^{\circ}\right)^{2}\right)$, Dataset 1

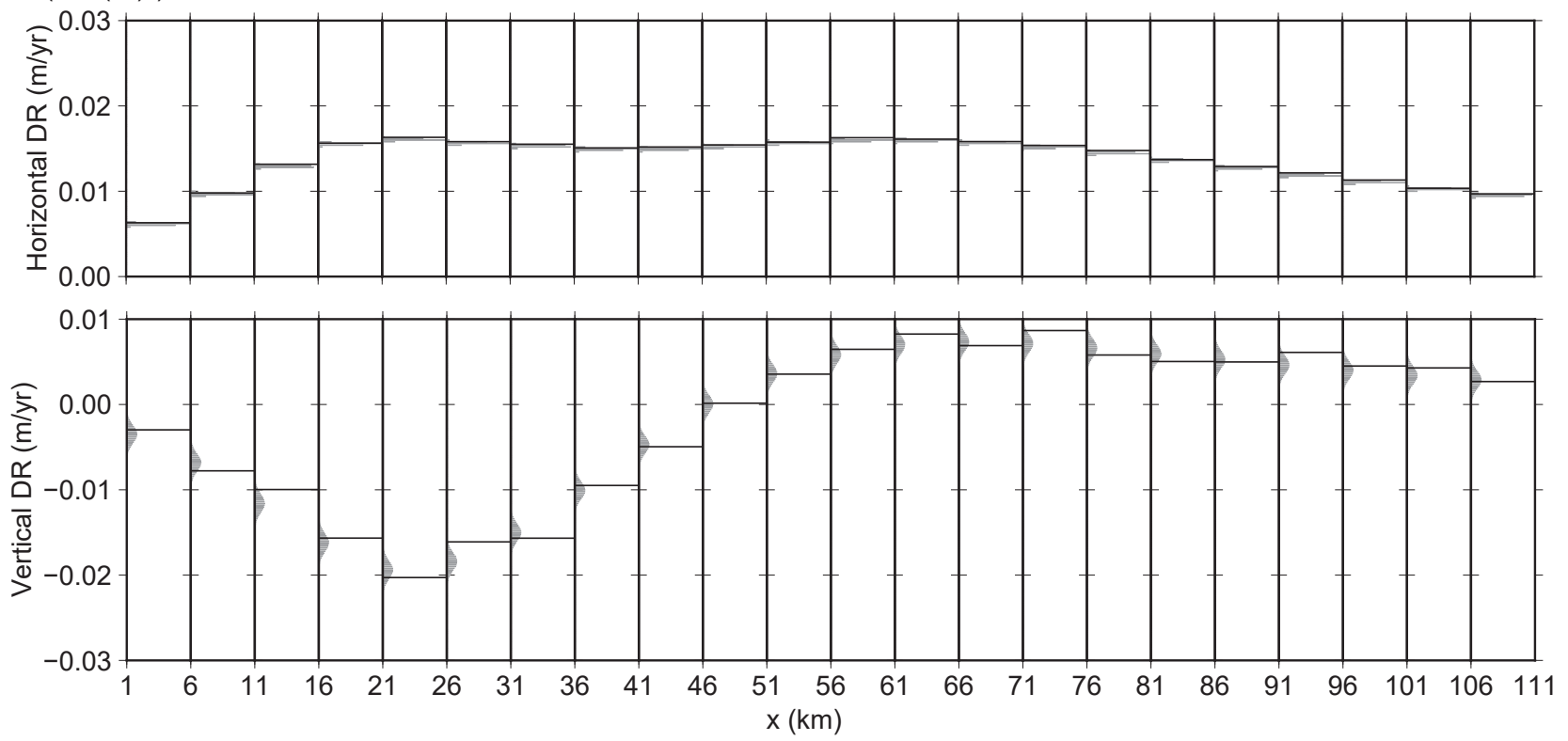

Figure 4. The posterior predicted PDF of horizontal and vertical displacement rate (DR) in the base case, using Dataset 1 and assuming that $\theta$ follows $\mathcal{N}\left(18^{\circ},\left(3^{\circ}\right)^{2}\right)$.

(a) $\mathcal{N}\left(12^{\circ},\left(3^{\circ}\right)^{2}\right)$, Dataset 1
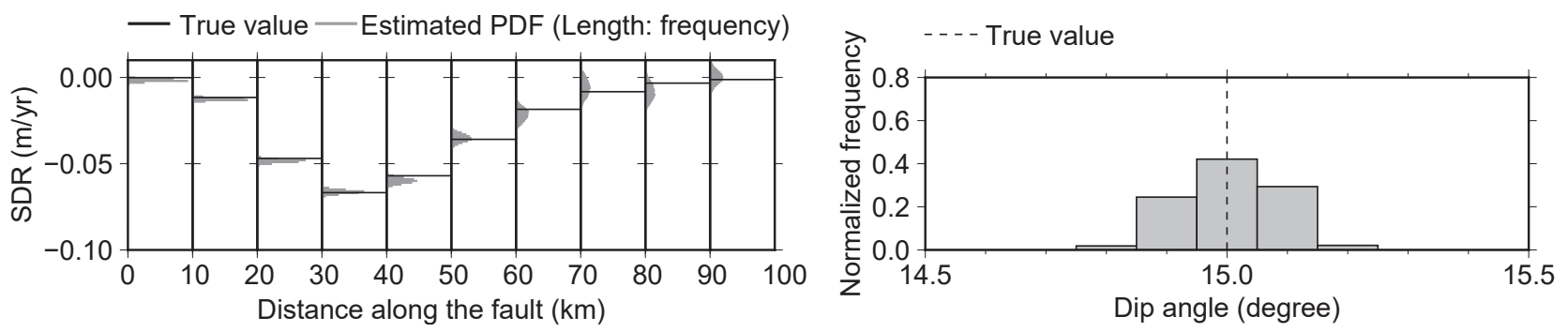

(b) $\theta=18^{\circ}$, Dataset 1

(c) $\theta=12^{\circ}$, Dataset 1
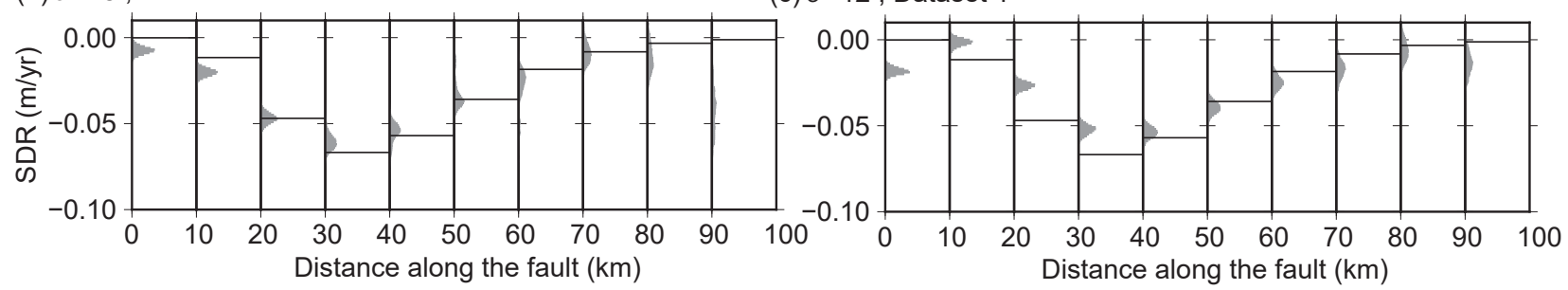

Figure 5. The estimation result based on a different assumption of the mean value of the dip angle and comparison with a conventional approach based on a smoothing constraint. (a) The estimation result of SDR and the dip angle in a case using Dataset 1 and assuming that $\theta$ follows $\mathcal{N}\left(12^{\circ},\left(3^{\circ}\right)^{2}\right)$. (b) The estimation result of SDR using a conventional approach based on a smoothing constraint and assuming $\theta=18^{\circ}$. (c) The estimation result of SDR using a conventional approach based on a smoothing constraint and assuming $\theta=12^{\circ}$. 


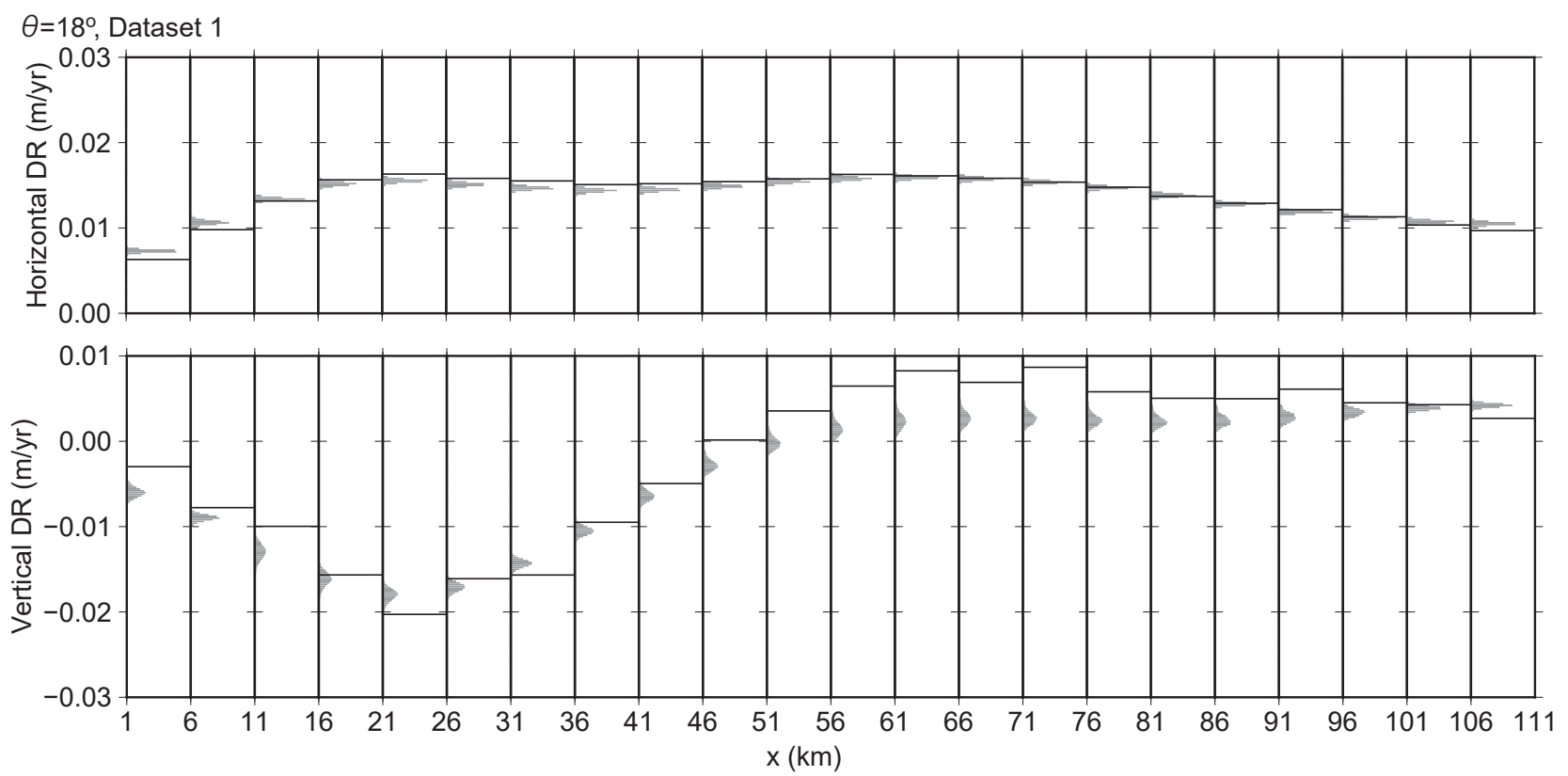

Figure 6. The posterior predicted PDF of horizontal and vertical displacement rate (DR) using a conventional approach based on a smoothing constraint and assuming $\theta=18^{\circ}$. 
(a) Case 1: $\mathcal{N}\left(18^{\circ},\left(3^{\circ}\right)^{2}\right)$, Dataset 2
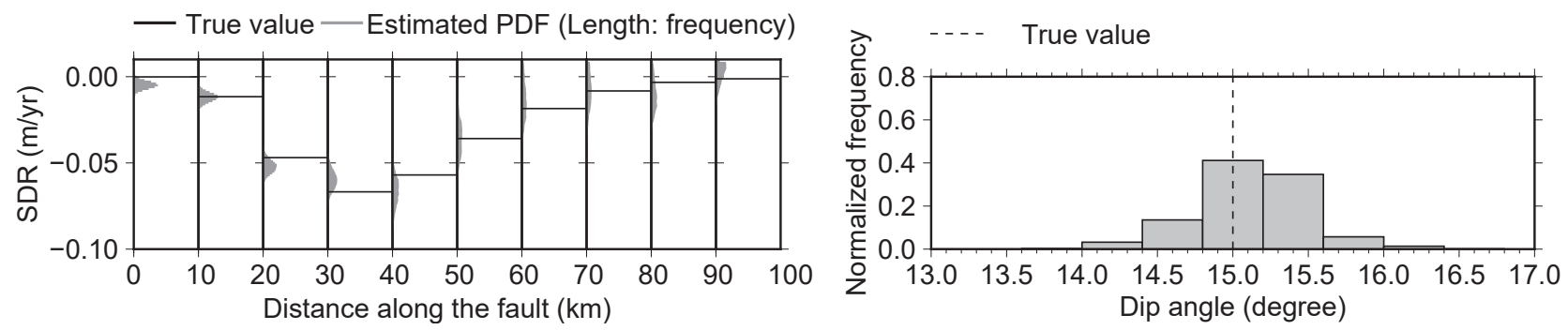

(b) Case 2: $\mathcal{N}\left(18^{\circ},\left(6^{\circ}\right)^{2}\right)$, Dataset 2
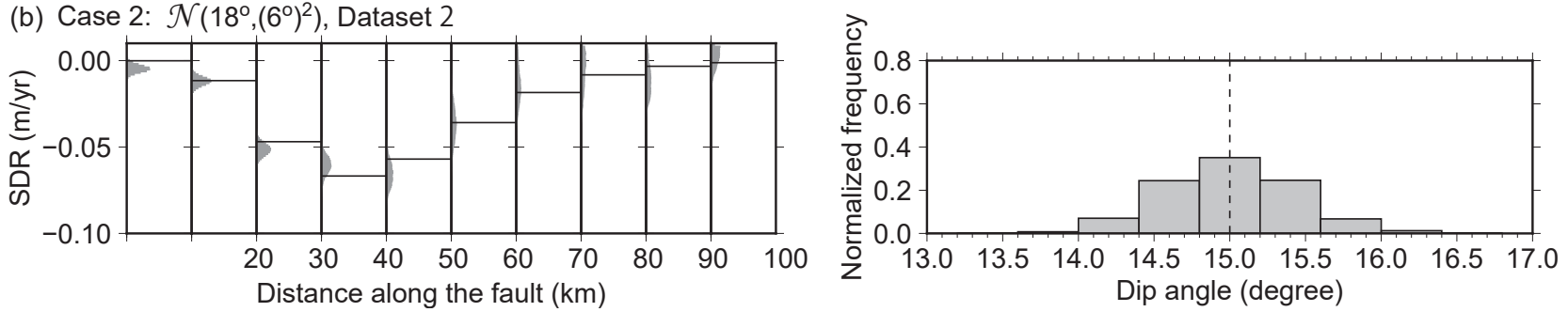

(c) Case 3: $\mathcal{N}\left(18^{\circ},\left(0.85^{\circ}\right)^{2}\right)$, Dataset 2
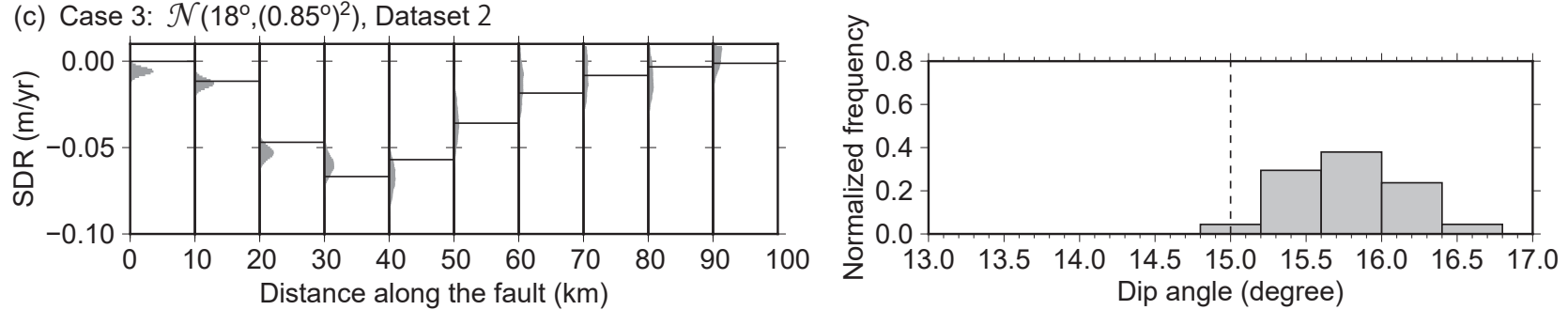

Figure 7. Comparison of the estimation results in Case 1, 2 and 3. (a) The estimation result of the posterior PDF of SDR and the dip angle in Case 1, using Dataset 2 and assuming that $\theta$ follows $\mathcal{N}\left(18^{\circ},\left(3^{\circ}\right)^{2}\right)$. (b) The estimation result of the posterior PDF of SDR and the dip angle in Case 2, assuming that $\theta$ follows $\mathcal{N}\left(18^{\circ},\left(6^{\circ}\right)^{2}\right)$. (c) The estimation result of the posterior PDF of SDR and the dip angle in Case 3 , assuming that $\theta$ follows $\mathcal{N}\left(18^{\circ},\left(0.85^{\circ}\right)^{2}\right)$. 
38 Non-peer reviewed manuscript submitted to Geophys. J. Int. by R. Agata

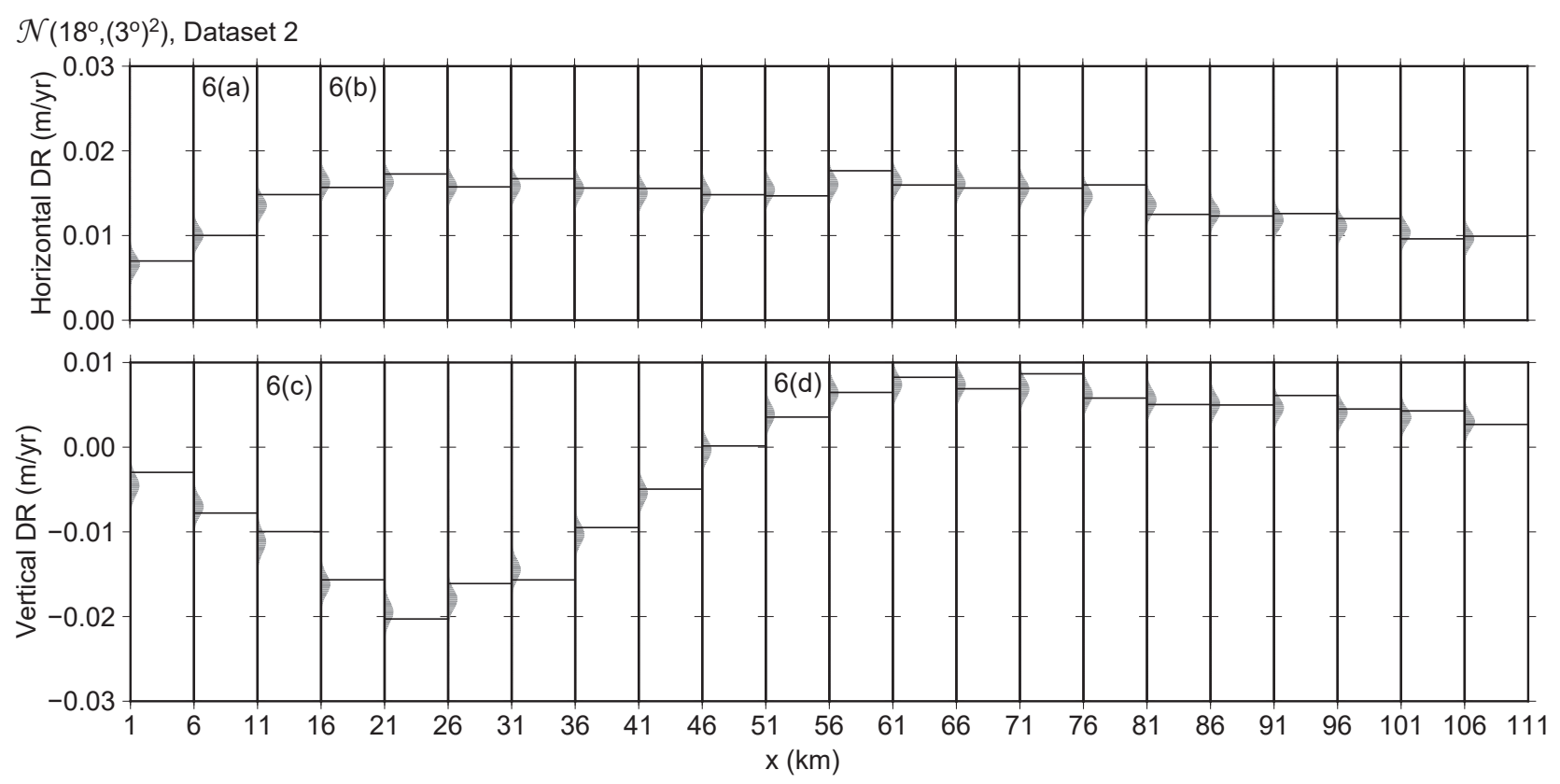

Figure 8. The posterior predicted PDF of horizontal and vertical displacement rate (DR) for Case 1. 

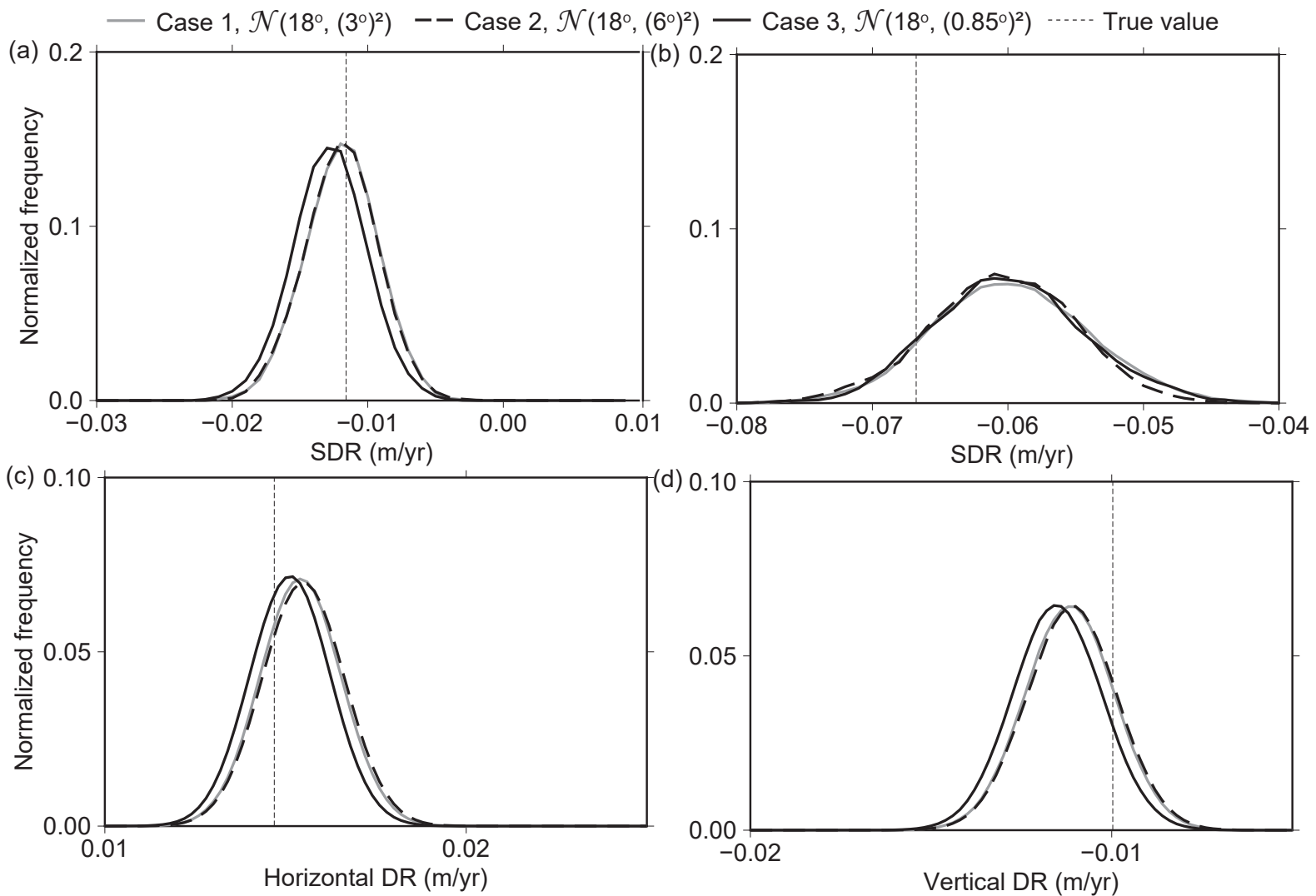

Figure 9. Close-up views of the samples of the posterior PDF for SDR and the posterior prediction PDF of Case 1, 2 and 3. (a)(b) Comparison of the posterior PDFs in the fault patches 6(a) and 6(b) marked in Figure 8. (c)(d) Comparison of the posterior prediction PDFs in the observation points 6(c) and 6(d) marked in Figure 8. 


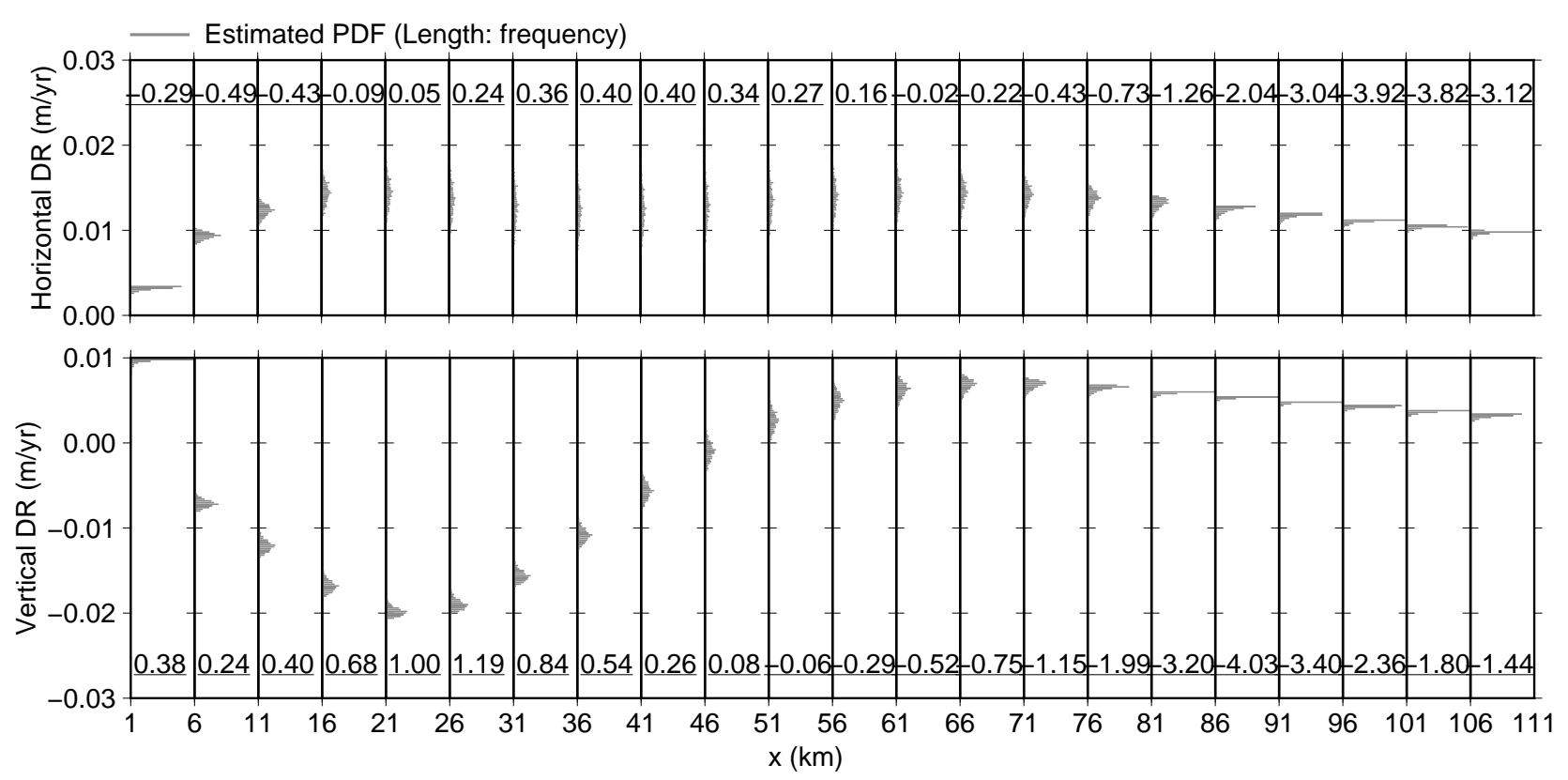

Figure 10. The prediction errors associated with the mean of the sampled model parameters $\overline{\mathbf{m}}$ in the base case. The underlined numbers are the skewness of the samples of the model parameter in each small fault.

(a) $\mathcal{N}\left(18^{\circ},\left(3^{\circ}\right)^{2}\right)$, Dataset $1, N=2000$

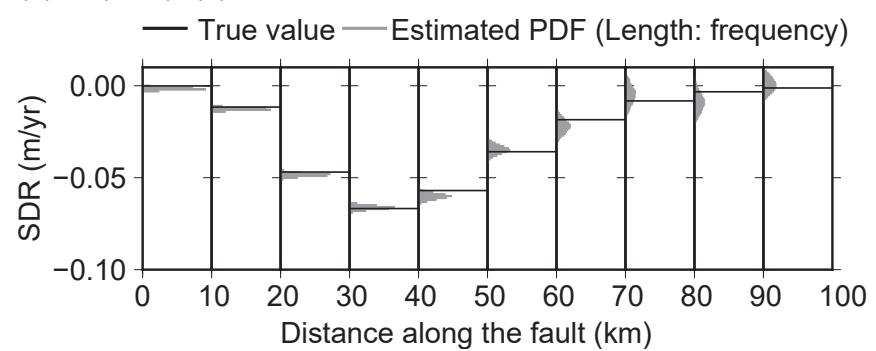

(b) $\mathcal{N}\left(18^{\circ},\left(0.85^{\circ}\right)^{2}\right)$, Dataset $2, N=1000$

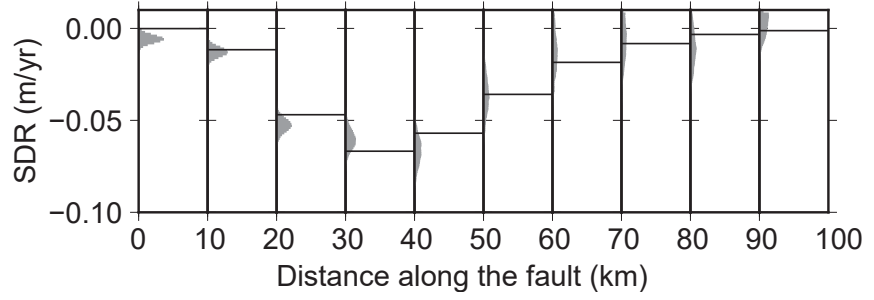

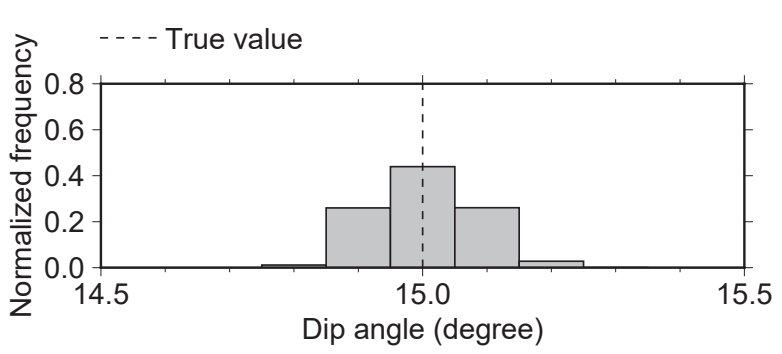

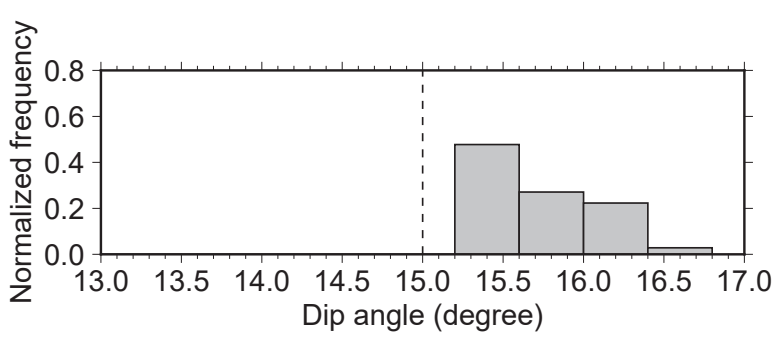

Figure 11. The estimation results with the ensemble sizes. (a) The estimation result of the posterior PDF of SDR and the dip angle with the ensemble size 2,000 for the same setting as the base case. (b) The estimation result of the posterior PDF of SDR and the dip angle with the ensemble size 1,000 for the same setting as Case 3. 


\section{APPENDIX A: FORMULATION OF A SLIP INVERSION ANALYSIS METHOD BASED ON THE SMOOTHING CONSTRAINT}

We present a slip estimation method that introduces regularization based on smoothing constraints and does not distinguish explicitly the prediction and observation errors, which is used in comparisons presented in Section 4.2.1. In the likelihood function in Equation 2, instead of considering the term of the prediction errors explicitly, the observation errors and prediction errors are considered without distinguishing each other in the form of Gaussian distribution, as

$p(\mathbf{d} \mid \mathbf{m}, \sigma)=(2 \pi)^{-N / 2}\left\|\sigma^{2} \mathbf{E}\right\|^{-1 / 2} \exp \left[-\frac{1}{2}(\mathbf{d}-\mathbf{G m})^{\mathrm{T}}\left(\sigma^{2} \mathbf{E}\right)^{-1}(\mathbf{d}-\mathbf{G m})\right]$,

Here we consider one of the most widely used approaches, where $\mathbf{E}$ is a diagonal matrix and does not have covariance components. We assume a prior PDF with respect to the smoothness of slip distribution,

$p\left(\mathbf{m} \mid \sigma_{\mathrm{p}}\right)=\left.(2 \pi)^{-P / 2}\left\|\frac{1}{\sigma_{\mathrm{p}}^{2}} \boldsymbol{\Lambda}\right\|\right|^{1 / 2} \exp \left[-\frac{1}{2} \mathbf{m}^{\mathrm{T}}\left(\frac{1}{\sigma_{\mathrm{p}}^{2}} \mathbf{L}^{\mathrm{T}} \mathbf{L}\right) \mathbf{m}\right]$,

where $\mathbf{L}$ is a matrix corresponding to a discretized Laplacian operator, $P$ is the $\operatorname{rank}$ of $\mathbf{L}^{\mathrm{T}} \mathbf{L}, \boldsymbol{\Lambda}$ is a $P \times P$ diagonal matrix that has non-zero eigenvalues of $\mathbf{L}^{\mathrm{T}} \mathbf{L}$ as its diagonal components, and $\sigma_{\mathrm{p}}$ is an unknown scaling factor. Based on Bayes' theorem, the posterior PDF of the model parameters is written as

$$
\begin{aligned}
p\left(\mathbf{m} \mid \mathbf{d}, \sigma, \sigma_{\mathrm{p}}\right) & =\kappa p(\mathbf{d} \mid \mathbf{m}, \sigma) p\left(\mathbf{m} \mid \sigma_{\mathrm{p}}\right) \\
& =\kappa(2 \pi)^{-(N+P) / 2}\left\|\sigma^{2} \mathbf{E}\right\|^{-1 / 2}\left\|\frac{1}{\sigma_{\mathrm{p}}^{2}} \mathbf{\Lambda}\right\|^{1 / 2} \exp \left[-\frac{1}{2} s(\mathbf{m})\right]
\end{aligned}
$$

where

$$
s(\mathbf{m})=(\mathbf{d}-\mathbf{G m})^{\mathrm{T}}\left(\sigma^{2} \mathbf{E}\right)^{-1}(\mathbf{d}-\mathbf{G m})+\mathbf{m}^{\mathrm{T}}\left(\frac{1}{\sigma_{\mathrm{p}}^{2}} \mathbf{L}^{\mathrm{T}} \mathbf{L}\right) \mathbf{m} .
$$

This formulation of the posterior PDF is identical to that presented in Yabuki \& Matsu'ura (1992). They introduced Akaike's Bayesian information criterion to determine the hyperparameters $\sigma$ and $\sigma_{\mathrm{p}}$. Instead, we here seek to draw samples from the joint posterior PDF of $\mathbf{m}, \sigma$ and $\sigma_{\mathrm{p}}$ using Algorithm 1, aiming for comparison in an equivalent condition to the proposed method. To formulate the joint posterior PDF, we introduce prior distributions for the hyperparameters, as $p\left(\mathbf{m}, \sigma, \sigma_{\mathrm{p}} \mid \mathbf{d}\right)=\kappa^{\prime} p(\mathbf{d} \mid \mathbf{m}, \sigma) p\left(\mathbf{m} \mid \sigma_{\mathrm{p}}\right) p(\sigma) p\left(\sigma_{\mathrm{p}}\right)$ 
42 Non-peer reviewed manuscript submitted to Geophys. J. Int. by R. Agata

We adopt

$p(\sigma)=p\left(\sigma_{\mathrm{p}}\right)=U(0, b)$,

where $\kappa^{\prime}$ is an updated normalization factor and $b$ is a sufficiently large number. Figure 5 (c) and (d) show the marginal posterior PDF for $\mathbf{m}$ obtained by integrating $p\left(\mathbf{m}, \sigma, \sigma_{\mathrm{p}} \mid \mathbf{d}\right)$ with $\sigma$ and $\sigma_{\mathrm{p}}$. 